\title{
Thermal fluctuations of hydrodynamic flows in nanochannels
}

\author{
François Detcheverry and Lydéric Bocquet \\ Institut Lumière Matière, UMR5306 Université Lyon 1-CNRS, Université de Lyon 69622 Villeurbanne, France
}

(Received 22 March 2013; published 8 July 2013)

\begin{abstract}
Flows at the nanoscale are subject to thermal fluctuations. In this work, we explore the consequences for a fluid confined within a channel of nanometric size. First, the phenomenon is illustrated on the basis of molecular dynamics simulations. The center of mass of the confined fluid is shown to perform a stochastic, non-Markovian motion, whose diffusion coefficient satisfies Einstein's relation, and which can be further characterized by the fluctuation relation. Next, we develop an analytical description of the thermally induced fluid motion. We compute the time- and space-dependent velocity correlation function, and characterize its dependence on the nanopore shape, size, and boundary slip at the surface. The experimental implications for mass and charge transports are discussed for two situations. For a particle confined within the nanopore, we show that the fluid fluctuating motion results in an enhanced diffusion. The second situation involves a charged nanopore in which fluid motion within the double layer induces a fluctuating electric current. We compute the corresponding contribution to the current power spectrum.
\end{abstract}

DOI: 10.1103/PhysRevE.88.012106

PACS number(s): 05.20.-y, 47.61.-k

\section{INTRODUCTION}

Transport processes in pores of nanometric size are ubiquitous in the living cell. While ionic channels control the passage of ions across the cell membrane [1], pores in the nucleus membrane facilitate the export of messenger RNA from the cell nucleus to the cytoplasm. The large family of aquaporins, found in the cell membrane of mammals, plants, and micro-organisms, enables rapid and selective water transport while excluding ionic species, most notably protons [2]. Aside from pores and channels that allow passive transport along a chemical potential gradient, active transport systems consuming metabolic energy permit motion against the gradient. All of those functions rely on molecular structures tailored at the nanoscale.

There is much interest in building artificial devices, which could replicate the size and function of biological channels. Such systems can be useful for a broad range of applications, including ultrafiltration, reverse osmosis, gas separation, or controlled drug delivery [3]. While reproducing the complex structures of biological pores remains presently out of reach, progress has been made recently in designing and fabricating nanometric pores. For instance, membranes for molecular separation, based on ultrathin porous nanocrystalline silicon or diamondlike carbon nanosheets [4,5] combine fast permeation and mechanical strength. New fabrication methods, such as ion beam sculpting, have been developed to fabricate single, nanometer-sized, solid-state nanopores [6,7], with application in macromolecule translocation for DNA sequencing [8-10]. Other prominent nanofluidic devices, with potential use in filtration, desalination, and energy conversion, are those built on nanotubes [11-13]. Because they seemingly allow for an extremely fast flow of water, carbon nanotubes could be the building block for a manmade analog to aquaporins, whereas the high surface charge of boron nitride nanotubes could open the way to efficient harvesting of osmotic power driven by salinity gradients [14].

All examples above, biological or synthetic, involve flows of water through conduits whose smallest size is in the nanometric range or below. When compared to their microfluidic counterparts $[15,16]$, those nanofluidic flows can be expected to differ in several respects [17-20]. First, the large surface to volume ratio makes surface phenomena predominant. Because interfacial effects occur on a characteristic length which becomes comparable to the system size (e.g., the Debye length for the electric double layer), they can not be subsumed into effective boundary conditions. Second, a coarse-grained, continuum description might fail and a molecularly detailed description becomes necessary. ${ }^{1}$ This is not only true for biological structures, whose behavior can be sensitive to precise arrangement of amino acids in the pore-forming protein complex, but can also apply to nonbiological systems. For instance, the low friction of water in carbon nanotubes involves incommensurability between the water and nanotube structures; other molecules of comparable size, but distinct shape, might lead to different behavior [22]. Third, even within a continuum modeling, nanoflows of molecular fluid may require, aside from the velocity field, the introduction of a new degree of freedom: the spin field. It describes the local angular velocity of rotating molecules, and couples to the translational degrees of freedom, as described by extended Navier-Stokes equations [23-25]. While irrelevant at the macroscopic scale, this intrinsic rotational momentum can play a role at the nanoscale, giving rise to unusual effects such as electrohydraulic power conversion. Finally, there is a new, generic, ingredient that will play a role in nanoflows: thermal fluctuations. They are the focus of this work.

It is a familiar idea that fluctuations play an increasingly important role as the system shrinks in size [26]. The finite-size effects that result in deviation from bulk quantities alter not only static quantities such as density, but also dynamic quantities, including transport coefficients. A significant impact of fluctuations is most expected in the limiting case of single-file regime, where extreme confinement constrains molecules to

\footnotetext{
${ }^{1}$ In particular, departure from the bulk properties might occur. For instance, the dielectric static permittivity of water near interfaces is not uniform [21].
} 


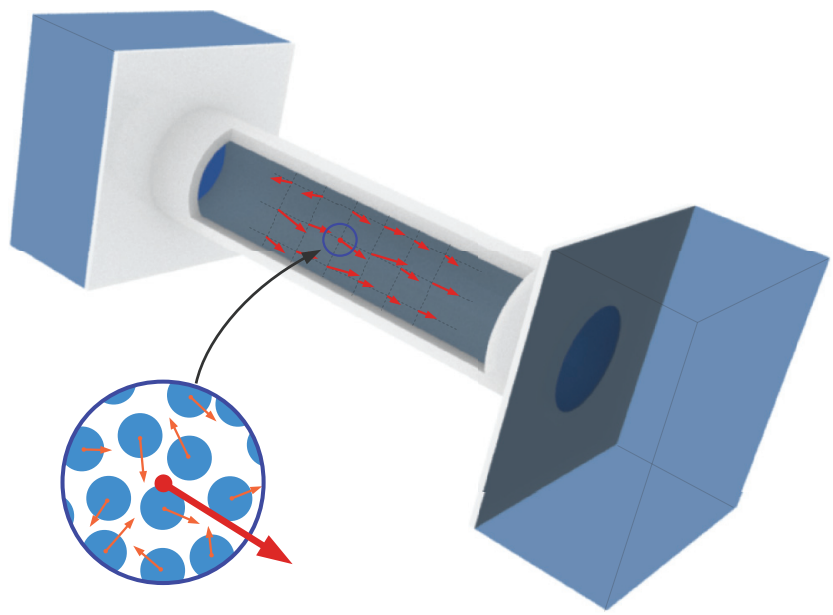

FIG. 1. (Color online) Schematics of a fluid confined in a nanochannel connecting two reservoirs. Thermal agitation of molecules (closeup) results in a stochastic, hydrodynamic flow.

unidimensional motion [27,28]. Yet, even in larger channels, for which the fluid can still be described as a continuum, pronounced effects of thermal agitation can be anticipated. An order of magnitude argument suggests that fluctuations become significant in systems of nanometric size [19]. This expectation is borne out by studies relying on fluctuating hydrodynamics, i.e., Navier-Stokes equation to which an additional random term is added to account for thermal noise. It turns out that fluctuations deeply impact the breakup of nanojets [29,30], as well as the spreading of droplets, for which the asymptotic behavior differs from Tanner's law [31]. In dewetting dynamics, thermal noise can accelerate the rupture of thin liquid films [32] and qualitatively alter the spectrum of capillary wave in films of polymers [33]. This series of results obtained for free-surface flows points to the relevance of fluctuations in nanoflows.

Perhaps surprisingly, the simpler but common situation of a fluid confined inside a nanopore has been left unstudied. In this work, we intend to fill this gap. The situation we consider is depicted in Fig. 1: a channel of nanometric size connecting two reservoirs filled with fluid. ${ }^{2}$ While at the Navier-Stokes level the fluid is at rest, the thermal agitation of molecules actually results in the stochastic flow of the fluid confined in the nanopore. This spontaneous motion is usually neglected in theoretical approaches, such as the PoissonNernst-Planck equation for ion dynamics [17], which are mean-field descriptions. Whereas the ions perform Brownian motion, the solvent, regarded as a dielectric medium and a thermal bath, has no degree of freedom of its own, and is therefore not subject to fluctuations. On the experimental

\footnotetext{
${ }^{2}$ It is customary to distinguish pores from channels by their aspect ratio, which is of order unity for the former and much larger for the latter. There is no clear-cut boundary, however, and in this work, both terms are employed. More generally, a conduit might be long enough to be called a channel if the "bulk" contribution, which originates inside the pore, dominates over the boundary contribution. The criterion, however, could depend on the phenomenon considered, e.g., friction for mass transport or conductance for charge transport.
}

side, the effect of noise on nanofluidic transport remains mostly unexplored. One recent study reported current noise in solid-state nanopores but focuses on optimizing detection efficiency for translocation events [34].

The study is organized as follows. Section II illustrates the phenomenon at work using molecular dynamics simulations. The center of mass of the confined fluid is shown to perform a non-Markovian stochastic motion, which can be characterized with the Einstein relation. A complementary view is given by the fluctuation relation and presented in Appendix A. In Sec. III, we develop an analytical approach that captures the thermally induced fluid motion, and compute the fluid velocity correlation function. Implications are discussed in Sec. IV. In particular, it is shown that a particle going through the channel can exhibit an enhanced diffusion due to fluid motion. Next, in Sec. V, we apply our results to the case of a charged nanopore and show that in the presence of a permanent charge distribution, the thermal fluctuations yield a new contribution to electric noise. Finally, a brief conclusion is given in Sec. VI. While a short account of this work was given in Ref. [35], here we present the method of derivation, consider both the slit and cylindrical channels, and give a full discussion of the results.

\section{MOLECULAR DYNAMICS SIMULATIONS}

Thermal fluctuations are de facto included in molecular dynamics (MD) simulations. As such, their effects have been noticed in previous studies, not only for single-file water flow in carbon nanotubes [27,36], but also for channels a few nanometers in size [37]. Here, we consider a simple fluid confined in a solid nanochannel and show that even if the average flow is well described by continuum hydrodynamics, the effect of thermal fluctuations is clearly visible.

\section{A. Model}

In MD simulations of flows, the fluid is often maintained at constant temperature with thermostats developed for equilibrium situations, even though the driving force makes the system out of equilibrium. This approach has proven adequate when the focus is on average quantities, such as the velocity profile of the stationary flow. In this work, we are interested not only in the average quantities, but also in the fluctuations and a particular attention must be paid to the thermostating method. To minimize any potential bias related to the thermostat, a natural idea is to thermostat not the fluid itself, but the solid that surrounds it, as actually happens in an experiment. This method was employed in a number of works (see, e.g., Refs. [38-41]), but only recently it has been given a sound basis and given specific prescription [42-44]. In those stochastic boundary conditions, a Langevin thermostat is applied only to the "border" particles, while the other particles evolve according to Newton's law. Here we take the solid particles as the "border."

The solid consists of a collection of independent oscillators: solid particles are tethered to an anchoring point by a harmonic spring, and do not interact with each other. As a consequence, the pseudocrystalline solid has no collective mode of vibration. While it would be interesting to study how the vibration modes of a particular solid can couple to the fluctuations of the fluid 


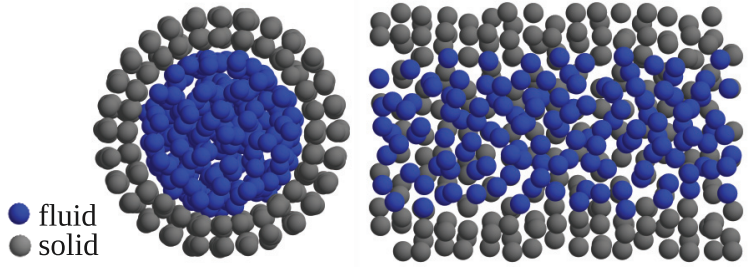

FIG. 2. (Color online) Cross-sectional snapshots of the simulation system.

motion, here we want to focus on the intrinsic dynamics of the fluid, in the absence of external influence. ${ }^{3}$ This minimal model of solid is sufficient to act as a thermostat.

All particles have equal mass. The fluid-fluid and fluid-solid pairs of particles interact through the same Lennard-Jones (LJ) potential. If not mentioned otherwise, quantities are given in LJ units. The solid used in simulation is shown in Fig. 2. The anchoring points are the nodes of two [100] fcc planes rolled up to form a tube with inner radius $R$ and length $L$. The spring constant $k=500$ is high enough for the Lindemann criterion to be satisfied. ${ }^{4}$ The oscillatory period is $\tau_{\text {osc }}=0.28$ and relaxation processes occurring on longer time scale can only be associated with the fluid. The solid particles are subject to a Langevin thermostat at temperature $T=2$, with a damping constant chosen so that oscillators are critically damped.

In the following, the channel has a radius $R=3.5$ and a length $L=12.7$. Assuming $\sigma=0.3 \mathrm{~nm}$, this would translate into real dimensions $R \simeq 1 \mathrm{~nm}$ and $L \simeq 4 \mathrm{~nm}$, and a volume $V \simeq 12 \mathrm{~nm}^{3}$. To induce a flow along the pore, the $N=275$ fluid particles can be subject to a gravity $g$. We set $g=0$ or 0.02 , the latter being low enough to remain in the regime of linear response. Boundary conditions along the pore $(x$ direction) are periodic; in this respect, the system is somewhat artificial when compared to a real experimental setup but it provides a simple setup for our investigation. Finally, the time step is set to $5 \times 10^{-4}$. All simulations were carried out using the LAMMPS package [45].

Before focusing on fluctuations, let us first characterize the average properties of the flow. For simplicity, radial profiles are used, even though the solid has only approximate radial symmetry. The density and velocity profiles under gravity are shown in Fig. 3. Within the range of gravity studied, the density profiles are independent of the velocity and are identical to the profile at equilibrium. One can picture the fluid as forming three concentric shells in the channel. As regards the temperature, it is uniform across the system (not

\footnotetext{
${ }^{3}$ Simulations with more realistic models have shown the influence of the solid. If, for instance, solid particles interact through a LJ potential, the velocity correlation function of the solid center of mass exhibits slowly damped oscillations, whose frequency is set by the spring constant. Such oscillations are also visible in the velocity correlation of the fluid center of mass.

${ }^{4}$ Denoting as $\left\langle\delta x^{2}\right\rangle$ the mean-square displacement of the solid particles from their lattice site, and $d$ the nearest-neighbor distance in the lattice, the Lindemann criterion states that the crystal does not melt as long as $\sqrt{\left\langle\delta x^{2}\right\rangle} / d<C_{L}$, where $C_{L}$ is a weakly material-dependent constant with typical value 0.15 .
}
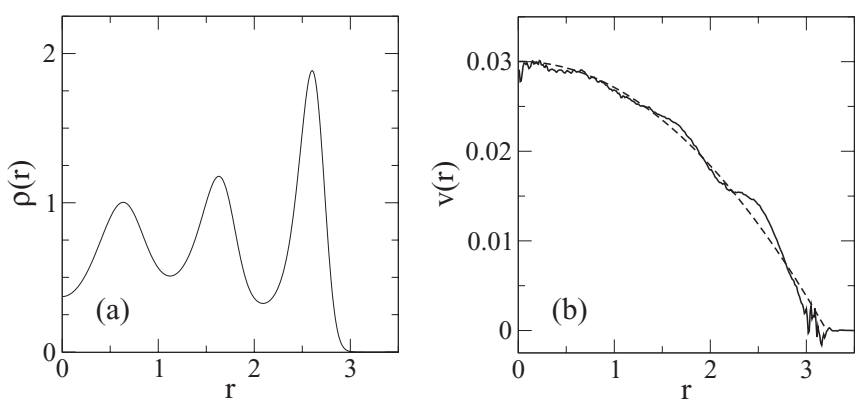

FIG. 3. (a) Average density profile. (b) Average velocity profile under gravity. The dashed line is a two-parameter fit to a parabola $v(r)=v_{\max }\left[1-(r / R)^{2}\right]$, that yields $R=3.21$ and $v_{\max }=0.030$.

shown). The velocity profile exhibits irregularities induced by the fluid structure, as observed previously [46], but remains approximately parabolic.

For a fluid of total mass $m$ and viscosity $\eta$ flowing under gravity $g$ in a cylindrical tube of length $L$ with no-slip boundary condition, macroscopic hydrodynamics predicts a parabolic velocity profile, with maximum velocity $v_{\max }=m g /(4 \pi \eta L)$. Let us assume that, in spite of the strong confinement, one can use the bulk viscosity of the LJ fluid, as parametrized in Ref. [47]. The viscosity depends sensitively on the average density, and thus on the radius taken for the cavity. Taking a radius $R=3.5$ yields $v_{\max }=0.021$ while taking $R=3$ yields $v_{\max }=0.044$. Those two cases bracket the value 0.030 observed in simulation. Taking $R=3.21$, the value obtained from a parabolic fit of the velocity profile (Fig. 3), one gets $v_{\max }=0.031$, quite close to the simulation result, showing that macroscopic hydrodynamics keeps some predictive power even in a nanometric channel. Although it may not always hold (see Ref. [48] for a recent counterexample and references therein), such remarkable robustness of the continuum description has been found previously $[46,49,50]$.

\section{B. Motion of the fluid center of mass}

As a simple indication of the fluid motion, the position of the fluid center of mass (c.m.) is shown in Fig. 4. It characterizes the collective motion of the fluid as a whole. The trajectories exhibit a back and forth motion typical of a random walk, with a drift controlled by $g$.
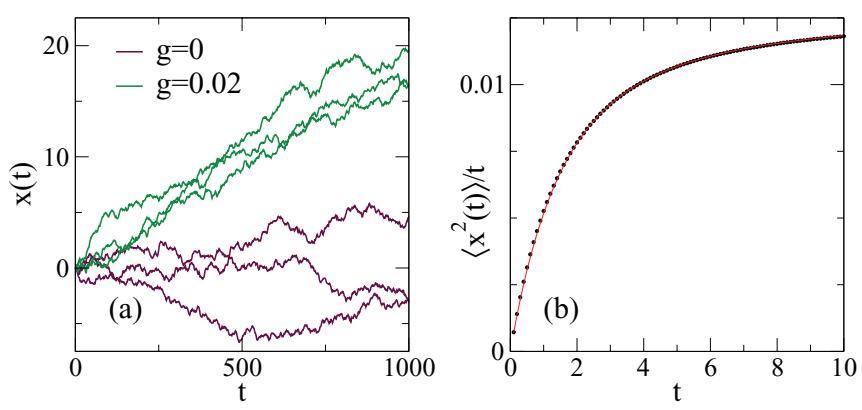

FIG. 4. (Color online) (a) Trajectories of the fluid c.m. with and without gravity (top and bottom, respectively), three simulations are shown in each case. (b) Mean-square displacement $\left\langle x^{2}(t)\right\rangle$ divided by time $t$, for $g=0$. The line is a one-parameter fit according to Eq. (1). 


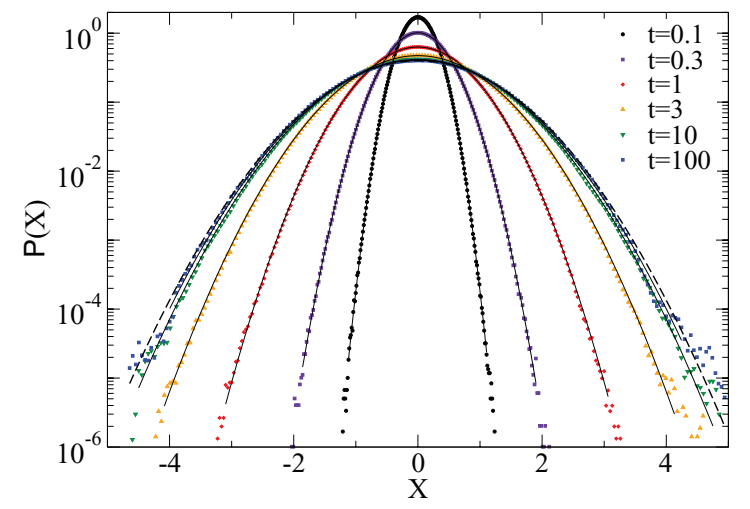

FIG. 5. (Color online) Probability distribution of the rescaled displacement $X=x(t) / \sqrt{2 D t}$ of the fluid c.m. Continuous lines are fits to a Gaussian distribution. The dashed line is a standard Gaussian.

Figure 5 plots the probability distribution for the rescaled displacement $x(t) /(2 D t)$. Consistent with the random walk, the distributions are identical at large time $(t \gtrsim 10)$ and collapse on the standard Gaussian. On shorter times, the distributions remain Gaussian, but with a deviation smaller than unity. Since the distributions have zero mean, they are completely characterized by the mean-square displacement $\left\langle x^{2}(t)\right\rangle$, which is plotted in Fig. 4. For a Brownian motion obeying the Langevin equation $\ddot{x}=-\gamma \dot{x}+F_{R}(t) / m$ with delta-correlated random force $F_{R}(t)$, the mean-square displacement is given by [51]

$$
\left\langle x^{2}(t)\right\rangle=2 D t\left[1-\frac{1-e^{-\gamma t}}{\gamma t}\right], \quad \gamma=\frac{k_{B} T}{D m},
$$

where $k_{B}$ is the Boltzmann constant. As visible in Fig. 4, a fit with Eq. (1) provides an excellent description of the data and yields for the diffusion coefficient $D=6.50 \times 10^{-3}$. Another estimate for $D$ can be computed from the velocity correlation at zero driving force (see below), which yields $D=\int_{0}^{\infty} C(t) d t=6.57 \times 10^{-3} .5$ Besides, one can determine the mobility $\mu=\bar{v} / F$ from the average drift velocity $\bar{v}$ of the fluid under an applied driving force; numerically, we obtain $\mu=3.25 \times 10^{-3}$. Comparing mobility and diffusion, one finds that within $1 \%$ accuracy the Einstein relation is satisfied.

If the c.m. motion was a simple Langevin process as assumed above, the velocity correlation function $C(t)$ would be a simple decaying exponential. ${ }^{6}$ That this is not the case can be seen in Fig. 6. If the decay is purely exponential at long times, significant departure is seen at early times $(t \lesssim 1)$, which suggests memory effects in the c.m. motion. ${ }^{7}$ To conclude this section, we have shown an example of a fluid confined

\footnotetext{
${ }^{5}$ The small discrepancy can be attributed to inaccuracy in the long time behavior of $C(t)$.

${ }^{6}$ Note that for a bulk fluid the total momentum is conserved since the fluid does not exchange momentum with the walls and therefore $C(t)$ is a constant.

${ }^{7}$ Doob's theorem states that a process which is Gaussian and Markovian has for correlation function a pure exponential. However, we have not shown that our process is Gaussian: only the one time distribution is plotted, not the multitime distributions.
}

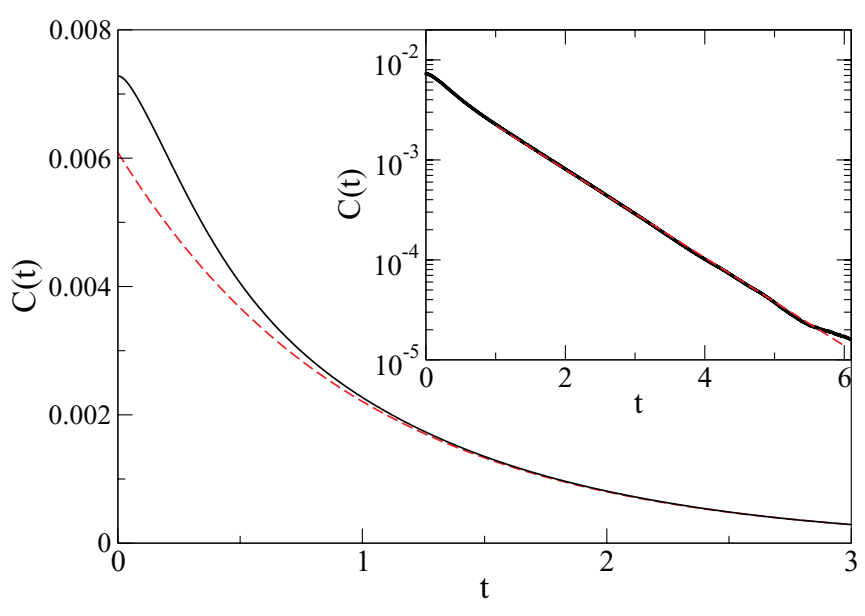

FIG. 6. (Color online) Correlation function of the c.m. velocity. The red (dashed) line is a fit to a simple exponential for $t \geqslant 1$. (Inset) Same data with logarithmic $y$ scale.

in a nanopore, whose average properties can be described by continuum hydrodynamics, but for which thermal fluctuations induce for the c.m. a stochastic, non-Markovian motion. As regards the c.m. motion, a complementary characterization is possible on the basis of the fluctuation relation; this is detailed in Appendix A.

\section{FLUCTUATIONS IN MASS TRANSPORT}

Having illustrated numerically the importance of thermal fluctuations on a confined nanoflow, we now develop an analytical understanding of those effects. To be generic and capture phenomena that are independent of molecular details, we choose a continuum description whose main variable is the velocity field. Our goal is to include thermal fluctuations in this description, and to obtain the time- and space-dependent velocity correlation function. We present three different routes to do so, which are equivalent but differ in their starting point: fluctuating hydrodynamics, regression hypothesis, and fluctuation-dissipation theorem. This section presents the derivation of the correlation function. Discussion of results is postponed to Sec. IV.

Let us first define our system, notations, and quantities of interest. We consider a periodic, parallelepipedic channel, with height $H$, width $W$, and length $L$. To keep calculation tractable, the flow is assumed to be unidirectional along the channel axis ( $x$ direction), that is, $\mathbf{v}(\mathbf{r}, t)=v(\mathbf{r}, t) \mathbf{e}_{x}$. The latter assumption is justified for long channel $L \gg H$ but is an approximation otherwise. While boundary conditions are periodic in the $x$ and $y$ directions, they include possible slippage at the channel walls, namely, $v=b \partial_{z} v$, where $b$ is the slip length, the distance within the solid where the velocity extrapolated linearly from the wall would vanish [19].

The basic quantity to characterize the fluid motion is the velocity correlation function

$$
C\left(z, z^{\prime}, t\right)=\left\langle u(z, t) u\left(z^{\prime}, 0\right)\right\rangle,
$$

where $u(z, t)$ is the average velocity of a layer at position $z$ :

$$
u(z, t)=\frac{1}{L W} \int_{0}^{L} \int_{0}^{W} v(\mathbf{r}, t) d y d x
$$


Knowing $C\left(z, z^{\prime}, t\right)$ gives access to several integrated quantities. The velocity of the fluid center of mass (c.m.) is

$$
\bar{u}(t)=\frac{1}{H} \int_{0}^{H} u(z, t) d z
$$

and the corresponding correlation function is

$$
C(t)=\langle\bar{u}(t) \bar{u}(0)\rangle=\frac{1}{H^{2}} \iint_{0}^{H} C\left(z, z^{\prime}, t\right) d z d z^{\prime} .
$$

We also introduce

$$
D\left(z, z^{\prime}\right)=\int_{0}^{\infty} C\left(z, z^{\prime}, t\right) d t .
$$

$D(z, z)$ is the diffusion coefficient of the fluid layer at position $z$. Finally, the diffusion coefficient for the fluid c.m. is

$$
D=\int_{0}^{\infty} C(t) d t
$$

Note that $D$ is defined in terms of the velocity correlation function of the c.m. and not from the individual velocities of fluid layers. ${ }^{8}$ Aside from the slit channel, the case of a cylindrical channel will also be addressed, with notations defined in the following.

\section{A. First route: Fluctuating hydrodynamics and solution for $C\left(z, z^{\prime}, t\right)$}

The fluctuating hydrodynamics (FH) approach supplements the Navier-Stokes equation with a random noise term that satisfies the fluctuation-dissipation theorem [52-55]. It has proven useful not only in the nanometric free-surface flows mentioned above, but also for computing properties of fluids that are at equilibrium or maintained out of equilibrium by a temperature or concentration gradient [56]. For an incompressible fluid of density $\rho$ and dynamic viscosity $\eta=\rho v$, FH equations for the velocity $\mathbf{v}$ read as

$$
\begin{gathered}
\nabla \cdot \mathbf{v}=0, \\
\rho\left[\partial_{t} \mathbf{v}+\mathbf{v} \cdot \nabla \mathbf{v}\right]=-\nabla p+\eta \Delta \mathbf{v}+\nabla \cdot S .
\end{gathered}
$$

$S$ is the random stress tensor, which is symmetric, of zero mean, and with correlations $\left\langle S_{i j}(\mathbf{r}, t) S_{k l}\left(\mathbf{r}^{\prime}, t^{\prime}\right)=2 \eta k_{B} T\left(\delta_{i j} \delta_{k l}+\right.\right.$ $\left.\delta_{i l} \delta_{j k}\right) \delta\left(\mathbf{r}-\mathbf{r}^{\prime}\right) \delta\left(t-t^{\prime}\right)$.

Given the simplifying assumptions given above, FH equations reduce to

$$
\partial_{t} u-v \partial_{z z} u=\zeta,
$$

where the noise term $\zeta$ is characterized by ${ }^{9}$

$$
\begin{aligned}
\langle\zeta(z, t)\rangle & =0, \\
\left\langle\zeta(z, t) \zeta\left(z^{\prime}, 0\right)\right\rangle & =\sigma_{\zeta} \partial_{z z^{\prime}} \delta\left(z-z^{\prime}\right) \delta(t), \quad \sigma_{\zeta}=\frac{2 \eta k_{B} T}{\rho^{2} L W} .
\end{aligned}
$$

\footnotetext{
${ }^{8} \mathrm{Or}$, in the case of a MD simulation, from the individual velocities of particles.

${ }^{9}$ As in Ref. [32], we assume that the components of the noise tensor scale like the corresponding component of the stress tensor, so only the term $\partial_{z} S_{x z}$ is kept.
}

Equations (9) and (10) define a diffusion equation with a random noise term and appear in the context of stochastic growth of interfaces, for the case of growth whose dynamics and noise are both conservative [57]. The similarity is incomplete, though, since our problem differs in the boundary conditions.

\section{Slit channel}

Let us introduce a set of functions $\left\{\psi_{n}\right\}$ that are solutions of the equation $\psi_{n}^{\prime \prime}=-\alpha_{n}^{2} \psi_{n}$ and satisfy prescribed boundary conditions for $z=0$ and $H$. Together, they form an orthonormal basis for the scalar product $f \cdot g=\int_{0}^{H} f(z) g(z) d z$, that is $\psi_{m} \cdot \psi_{n}=\delta_{m n}$. The solution is expanded on this basis as

$$
u(z, t)=\sum_{n=0}^{\infty} u_{n}(t) \psi_{n}(z),
$$

where each coefficient $u_{n}=\psi_{n} \cdot u$ obeys the Langevin equation

$$
\frac{d}{d t} u_{n}(t)=-v \alpha_{n}^{2} u_{n}(t)+\zeta_{n}(t) .
$$

The solution, once the initial conditions are "forgotten," reads as

$$
u_{n}(t)=\int_{-\infty}^{t} e^{-v \alpha_{n}^{2}\left(t-t^{\prime}\right)} \zeta_{n}\left(t^{\prime}\right) d t^{\prime} .
$$

Using the noise property $\left\langle\zeta_{m}(t) \zeta_{n}(0)\right\rangle=\sigma_{\zeta} \alpha_{n}^{2} \delta_{m n} \delta(t)$ yields

$$
\left\langle u_{m}(t) u_{n}(0)\right\rangle=\frac{\sigma_{\zeta}}{2 v} \delta_{m n} e^{-\nu \alpha_{n}^{2}|t|},
$$

and the velocity correlation function is then given by

$$
C\left(z, z^{\prime}, t\right)=\frac{\sigma_{\zeta}}{2 v} \sum_{n=0}^{\infty} \psi_{n}(z) \psi_{n}\left(z^{\prime}\right) e^{-\nu \alpha_{n}^{2}|t|} .
$$

Denoting by 1 the function equal to unity, the integrated quantities read as

$$
\begin{gathered}
C(t)=\frac{\sigma_{\zeta}}{2 v H^{2}} \sum_{n=0}^{\infty}\left(\psi_{n} \cdot 1\right)^{2} e^{-\nu \alpha_{n}^{2}|t|}, \\
D\left(z, z^{\prime}\right)=\frac{\sigma_{\zeta}}{2 v^{2}} \sum_{n=0}^{\infty} \frac{\psi_{n}(z) \psi_{n}\left(z^{\prime}\right)}{\alpha_{n}^{2}}, \\
D=\frac{\sigma_{\zeta}}{2 v^{2} H^{2}} \sum_{n=0}^{\infty} \frac{\left(\psi_{n} \cdot 1\right)^{2}}{\alpha_{n}^{2}} .
\end{gathered}
$$

Let us now apply those formulas. For the symmetric slip boundary conditions

$$
\begin{gathered}
u(0, t)=+\left.b \quad \partial_{z} u(z, t)\right|_{z=0}, \\
u(H, t)=-\left.b \partial_{z} u(z, t)\right|_{z=H},
\end{gathered}
$$

the basis functions read as

$$
\psi_{n}(z)=C_{n}\left[\sin \left(\alpha_{n} z\right)+b \alpha_{n} \cos \left(\alpha_{n} z\right)\right],
$$

where $\alpha_{n}$ is solution of $\tan \left(H \alpha_{n}\right)=2 b \alpha_{n} /\left(b^{2} \alpha_{n}^{2}-1\right)$ and $C_{n}^{-2}=\left(H+2 b+H b^{2} \alpha_{n}^{2}\right) / 2$. No explicit expression for $\alpha_{n}$ is available, and the infinite sum could not be performed in the general case. 
For no-slip boundary condition, the eigenvalues $\alpha_{n}$ are known

$$
\psi_{n}(z)=\sqrt{\frac{2}{H}} \sin \left(\alpha_{n} z\right), \quad \alpha_{n}=n \frac{\pi}{H},
$$

resulting in explicit expressions

$$
\begin{gathered}
C\left(z, z^{\prime}, t\right)=\frac{\sigma_{\zeta}}{v H} \sum_{n=1}^{\infty} \sin \left(n \pi \frac{z}{H}\right) \sin \left(n \pi \frac{z^{\prime}}{H}\right) e^{-v \pi^{2} n^{2} t / H^{2}}, \\
C(t)=\frac{\sigma_{\zeta}}{\pi^{2} v H} \sum_{\text {odd } n=1}^{\infty} \frac{e^{-v \pi^{2} n^{2} t / H^{2}}}{n^{2}}
\end{gathered}
$$

Besides, the infinite sum can be performed for $D\left(z, z^{\prime}\right)$. Using the identity

$$
\sum_{n=1}^{\infty} \frac{\cos (n u)}{n^{2}}=\frac{\pi^{2}}{6}-\frac{\pi}{2}|u|+\frac{u^{2}}{4} \quad \text { for }|u|<2 \pi
$$

one gets

$$
D\left(z, z^{\prime}\right)=\frac{k_{B} T}{\eta L W} \frac{z\left(H-z^{\prime}\right)}{H}, \quad z<z^{\prime} .
$$

For $z^{\prime}<z, z$ and $z^{\prime}$ must be interchanged. From Eq. (6), one finally obtains the diffusion coefficient ${ }^{10}$

$$
D=\frac{k_{B} T}{12 \eta} \frac{H}{L W} \text {. }
$$

Two side remarks are in order. First, equations on $C\left(z, z^{\prime}, t\right)$ are also the equations governing the dynamics of a polymeric chain in the Rouse model. ${ }^{11}$ Second, taking the limit $H \rightarrow \infty$ in Eq. (17), one can show that $C(t) \sim t^{-3 / 2}$, i.e., one recovers the long time tail in velocity autocorrelation function [59-61].

\section{Cylindrical channel}

The channel is now a cylinder of radius $R$ and length $L$. We consider the average velocity in a layer at radius $r$ :

$$
u(r, t)=\frac{1}{2 \pi L} \int_{0}^{L} \int_{0}^{2 \pi} v(\mathbf{r}, t) d \theta d x .
$$

All notations are similar to those introduced for the slit case, except that integrals $\int_{0}^{H} d z$ are replaced with $\int_{0}^{R} r d r, \partial_{z z}$ is replaced with $\Delta_{r}=\frac{1}{r} \partial_{r}\left(r \partial_{r}\right)$ and $\sigma_{\zeta}=2 \eta k_{B} T /\left(2 \pi \rho^{2} L\right)$. For the slip boundary condition $u(R, t)=-\left.b \partial_{r} u(r, t)\right|_{r=R}$, one gets

$$
C\left(r, r^{\prime}, t\right)=\frac{\sigma_{\zeta}}{v R^{2}} \sum_{n=1}^{\infty} \frac{J_{0}\left(\alpha_{n} r\right) J_{0}\left(\alpha_{n} r^{\prime}\right)}{J_{0}^{2}\left(\alpha_{n} R\right)+J_{1}^{2}\left(\alpha_{n} R\right)} e^{-v \alpha_{n}^{2} t} .
$$

$J_{p}$ is the Bessel function of the first kind of order $p$ and the $\alpha_{n}$ are the positive roots of

$$
J_{0}(\alpha R)-b \alpha J_{1}(\alpha R)=0 .
$$

\footnotetext{
${ }^{10} \mathrm{D}$ can also be obtained directly from Eq. (15) using the identity $\sum_{n=1}^{\infty} 1 / n^{4}=\pi^{4} / 90$.

${ }^{11}$ Indeed, $u(z, t)$ can be identified with $R_{j}(t)$, the position of the $j$ th bead in the chain [58]. Equation (17) gives the end-to-end vector correlation function $\left\langle R_{j}(t) R_{j}(0)\right\rangle$.
}

From Eq. (20), one derives

$$
C(t)=\frac{4 \sigma_{\zeta}}{v R^{4}} \sum_{n=1}^{\infty} \frac{e^{-v \alpha_{n}^{2} t}}{\alpha_{n}^{2}\left(1+b^{2} \alpha_{n}^{2}\right)} .
$$

\section{B. Second route: Regression hypothesis and solution for $\tilde{C}\left(z, z^{\prime}, \omega\right)$}

Here, we show that the equation on $C\left(z, z^{\prime}, t\right)$ can be directly written from the regression hypothesis of Onsager. This approach was used in Ref. [62] and we extend those results to slit with arbitrary slip length and channel with cylindrical geometry. Instead of obtaining $\tilde{C}\left(z, z^{\prime}, t\right)$ as a series, compact and explicit expressions are obtained for its time Laplace transform.

\section{Slit channel}

The Onsager hypothesis makes the connection between the regression of spontaneous microscopic fluctuations in a system close to equilibrium, and the relaxation of macroscopic variables in a nonequilibrium situation, by assuming that both are governed by the same law [63]. In the case at hand, the macroscopic (or average) velocity $\langle u(z, t)\rangle$ obeys the diffusion equation

$$
\partial_{t}\langle u\rangle-v \partial_{z z}\langle u\rangle=0,
$$

and satisfies slip boundary conditions. The regression hypothesis states that the correlation function $C\left(z, z^{\prime}, t\right)$ obeys the same equation

$$
\partial_{t} C\left(z, z^{\prime}, t\right)-v \partial_{z z} C\left(z, z^{\prime}, t\right)=0
$$

and satisfies the same boundary conditions. To obtain the initial condition, the fluid is assumed to be at equilibrium in the canonical ensemble, for which

$$
C\left(z, z^{\prime}, 0\right)=\sigma_{u} \delta\left(z-z^{\prime}\right), \quad \sigma_{u}=\frac{k_{B} T}{\rho L W} .
$$

Note that $C\left(z, z^{\prime}, t\right)$ is actually the fundamental solution of the diffusion equation and, as such, it appears in the study of heat conduction [64]. In this context, the slip boundary condition corresponds to the radiating boundary condition.

The solution to Eqs. (22) and (23) has been given above. We now introduce $\tilde{C}\left(z, z^{\prime}, \omega\right)$, the Laplace transform of $C\left(z, z^{\prime}, t\right)$ with respect to time

$$
\tilde{C}\left(z, z^{\prime}, \omega\right)=\int_{0}^{\infty} C\left(z, z^{\prime}, t\right) e^{-\omega t} d t,
$$

$\tilde{C}$ is defined by the boundary value problem

$$
\begin{aligned}
\left(\omega-v \partial_{z z}\right) \tilde{C}\left(z, z^{\prime}, \omega\right) & =\sigma_{u} \delta\left(z-z^{\prime}\right), \\
\tilde{C}\left(0, z^{\prime}, \omega\right) & =+\left.b_{0} \partial_{z} \tilde{C}\left(z, z^{\prime}, \omega\right)\right|_{z=0}, \\
\tilde{C}\left(H, z^{\prime}, \omega\right) & =-\left.b_{H} \partial_{z} \tilde{C}\left(z, z^{\prime}, \omega\right)\right|_{z=H},
\end{aligned}
$$

where we assume slip lengths $b_{0}$ and $b_{H}$ for the bottom and top walls, respectively. The solution is

$$
\tilde{C}\left(z, z^{\prime}, \omega\right)=\frac{k_{B} T}{\eta L W} \frac{\xi}{2 \Gamma(\xi)} f\left(z, z^{\prime}, \xi\right), \quad \xi=\sqrt{\frac{\nu}{\omega}},
$$


where we have defined

$$
\begin{aligned}
f\left(z, z^{\prime}, \xi\right)= & \left(\frac{b_{0} b_{H}}{\xi^{2}}+1\right) \cosh \left(\frac{\left|z-z^{\prime}\right|-H}{\xi}\right) \\
& +\left(\frac{b_{0} b_{H}}{\xi^{2}}-1\right) \cosh \left(\frac{\left|z+z^{\prime}\right|-H}{\xi}\right) \\
& -\frac{b_{0}+b_{H}}{\xi} \quad \sinh \left(\frac{\left|z-z^{\prime}\right|-H}{\xi}\right) \\
& -\frac{b_{0}-b_{H}}{\xi} \sinh \left(\frac{\left|z+z^{\prime}\right|-H}{\xi}\right), \\
\Gamma(\xi)= & \left(\frac{b_{0} b_{H}}{\xi^{2}}+1\right) \sinh (H / \xi)+\frac{b_{0}+b_{H}}{\xi} \cosh (H / \xi) .
\end{aligned}
$$

For $x<x^{\prime}, f\left(x, x^{\prime}, \xi\right)$ can be factorized as

$$
\begin{aligned}
f\left(z, z^{\prime}, \xi\right)= & 2\left[\sinh \left(\frac{z}{\xi}\right)+\frac{b_{0}}{\xi} \cosh \left(\frac{z}{\xi}\right)\right] \\
& \times\left[\sinh \left(\frac{H-z^{\prime}}{\xi}\right)+\frac{b_{H}}{\xi} \cosh \left(\frac{H-z^{\prime}}{\xi}\right)\right],
\end{aligned}
$$

which shows that $f\left(z, z^{\prime}, \xi\right)$ satisfies the boundary conditions given above and that the regular part of $\partial_{z z} f$ is $\omega / v f$. The nonregular term in $\partial_{z z} f$ is $-2 \Gamma(\xi) / \xi \delta\left(z-z^{\prime}\right)$.

Equation (25) applied to the symmetric case $b_{0}=b_{H}=b$ can be further simplified in the two limits of zero and infinite slip:

$$
\begin{aligned}
\tilde{C}\left(z, z^{\prime}, \omega\right)= & \frac{k_{B} T}{\eta L W} \frac{\xi}{2 \sinh (H / \xi)}\left[\cosh \left(\frac{\left|z-z^{\prime}\right|-H}{\xi}\right)\right. \\
& \left.+\kappa \cosh \left(\frac{\left|z+z^{\prime}\right|-H}{\xi}\right)\right]
\end{aligned}
$$

where $\kappa=-1$ and 1 for $b=0$ and $1 / b \rightarrow 0$, respectively, ${ }^{12}$ in agreement with Ref. [62].

The quantities $\tilde{C}(\omega), D\left(z, z^{\prime}\right)$, and $D$ can now be derived from $\tilde{C}\left(z, z^{\prime}, \omega\right)$. Introducing the Womersley number $\epsilon=$ $H / \xi=H \sqrt{\omega / \nu},{ }^{13}$ the result reads as

$$
\begin{aligned}
\tilde{C}(\omega) & =\frac{k_{B} T}{m \omega}\left[1+\frac{2[1-\cosh (\epsilon)]-\frac{b_{0}+b_{H}}{H} \epsilon \sinh (\epsilon)}{\epsilon \Gamma(\epsilon)}\right], \\
\Gamma(\epsilon) & =\left(\frac{b_{0} b_{H}}{H^{2}} \epsilon^{2}+1\right) \sinh (\epsilon)+\frac{b_{0}+b_{H}}{H} \epsilon \cosh (\epsilon) .
\end{aligned}
$$

In the symmetric case $b_{0}=b_{H}=b, \tilde{C}(\omega)$ takes a simpler form

$$
\tilde{C}(\omega)=\frac{k_{B} T}{m \omega}\left[1-\frac{2 / \epsilon}{\operatorname{cotanh}\left(\frac{\epsilon}{2}\right)+\frac{b}{H} \epsilon}\right] .
$$

$D\left(z, z^{\prime}\right)$ is obtained from $\tilde{C}\left(z, z^{\prime}, \omega\right)$ by taking the limit $\omega \rightarrow$ 0 , which gives

$$
D\left(z, z^{\prime}\right)=\frac{k_{B} T}{\eta L W} \frac{\left(z+b_{0}\right)\left(H+b_{H}-z^{\prime}\right)}{H+b_{0}+b_{H}}, \quad z<z^{\prime} .
$$

\footnotetext{
${ }^{12}$ For the case $b=\infty$, the solution includes an additional constant term. Indeed, the initial condition (23) is not valid any more due to conservation of the total momentum [62].

${ }^{13}$ Introduced in the context of arterial flows, the Womersley number compares the forcing pulsation $\omega$ to the time required to adjust a viscous flow, i.e., for the momentum to diffuse over the channel [65].
}

$D\left(z, z^{\prime}\right)$ can also be found directly by solving the equation

$$
\partial_{z z} D\left(z, z^{\prime}\right)=-\frac{k_{B} T}{\eta L W} \delta\left(z-z^{\prime}\right) .
$$

Finally, the diffusion coefficient for the fluid center of mass is obtained by spatial integration of $D\left(z, z^{\prime}\right)$ or by computing $\lim _{\omega \rightarrow 0} \tilde{C}(\omega)$. Both routes lead to

$$
D=\frac{1}{12}\left(1+3 \frac{b_{0}+b_{H}+4 \frac{b_{0} b_{H}}{H}}{H+b_{0}+b_{H}}\right) \frac{k_{B} T}{\eta} \frac{H}{L W} .
$$

For the symmetric case, the term in brackets simplifies to $1+6 b / H$.

\section{Cylindrical channel}

The regression hypothesis now yields the equations

$$
\begin{aligned}
\left(\omega-v \Delta_{r}\right) \tilde{C}\left(r, r^{\prime}, \omega\right) & =\frac{\sigma_{u}}{r} \delta\left(r-r^{\prime}\right), \quad \sigma_{u}=\frac{k_{B} T}{2 \pi \rho L} \\
\tilde{C}\left(R, r^{\prime}, \omega\right) & =-\left.b \partial_{r} \tilde{C}\left(r, r^{\prime}, \omega\right)\right|_{r=R},
\end{aligned}
$$

whose solution reads as

$$
\begin{aligned}
\tilde{C}\left(r, r^{\prime}, \omega\right) & =\frac{k_{B} T}{2 \pi \eta L} f\left(r, r^{\prime}, \xi\right), \quad \xi=R \sqrt{\frac{\omega}{v}} \\
f\left(r, r^{\prime}, \xi\right) & =I_{0}\left(\frac{r}{\xi}\right)\left[\Gamma(\xi) I_{0}\left(\frac{r^{\prime}}{\xi}\right)+K_{0}\left(\frac{r^{\prime}}{\xi}\right)\right], \\
\Gamma(\xi) & =\frac{-K_{0}+\frac{b}{\xi} K_{1}}{I_{0}+\frac{b}{\xi} I_{1}}\left(\frac{R}{\xi}\right) .
\end{aligned}
$$

Here, $I_{p}$ and $K_{p}$ are the modified Bessel functions of order $p$ of the first and second kinds, respectively. Equation (31) applies for $r<r^{\prime}$, otherwise $r$ and $r^{\prime}$ should be interchanged. For the c.m. correlation, one gets

$$
\tilde{C}(\omega)=\frac{k_{B} T}{m \omega}\left[1-\frac{2 I_{1}(\epsilon) / \epsilon}{I_{0}(\epsilon)+\frac{b}{R} \epsilon I_{1}(\epsilon)}\right], \quad \epsilon=\frac{R}{\xi}
$$

and for $D\left(r, r^{\prime}\right)$,

$$
D\left(r, r^{\prime}\right)=\frac{k_{B} T}{2 \pi \eta L}\left[-\ln \frac{\max \left(r, r^{\prime}\right)}{R}+\frac{b}{R}\right],
$$

which is the solution of

$$
\Delta_{r} D\left(r, r^{\prime}\right)=-\frac{k_{B} T}{2 \pi \eta L} \frac{\delta\left(r-r^{\prime}\right)}{r} .
$$

Finally, the c.m. diffusion coefficient is

$$
D=\frac{1}{8 \pi}\left[1+\frac{4 b}{R}\right] \frac{k_{B} T}{\eta L} .
$$

\section{Third route: Fluctuation-dissipation theorem}

In Sec. III A, we started from the general equations of fluctuating hydrodynamics, whose random stress tensor is chosen to satisfy the fluctuation-dissipation theorem (FDT). An alternative route is to apply the FDT directly to the situation of interest. For simplicity, the method is illustrated with the slit geometry and the no-slip boundary condition.

The simplest way to compute $\tilde{C}(\omega)$ is to consider the motion of the fluid c.m. and apply the first fluctuation-dissipation 
theorem [51]

$$
\mu(\omega)=\frac{1}{k_{B} T} \int_{0}^{\infty}\langle\bar{u}(t+\tau) \bar{u}(t)\rangle e^{i \omega \tau} d \tau,
$$

where $\mu(\omega)$ is the complex admittance (mobility) defined by $\langle\bar{u}\rangle=\mu(\omega) F(\omega)$, and $F(\omega)$ is a periodic force applied on the fluid. Taking $F(\omega)=F e^{-i \omega t}$, where $F$ is the total force acting on the fluid, the resulting periodic flow is

$$
u(z, \omega)=\mu(z, \omega) F=\frac{i g}{\omega}\left[1-\frac{\cosh [\alpha(z-H / 2)]}{\cosh (\alpha H / 2)}\right],
$$

where $\alpha=\sqrt{-i} / \xi=\sqrt{-i \omega / v}$. The "mobility" for the c.m. is therefore

$$
\mu(\omega)=\frac{1}{H} \int_{0}^{H} \mu(z, \omega) d z=\frac{i}{m \omega}\left[1-\frac{2}{\alpha H} \tanh (\alpha H / 2)\right] .
$$

With our notations, Eq. (34) reads as $\tilde{C}(\omega)=k_{B} T \mu(i \omega)$, that is,

$$
\tilde{C}(\omega)=\frac{k_{B} T}{m \omega}\left[1-\frac{2}{\epsilon} \tanh \left(\frac{\epsilon}{2}\right)\right], \quad \epsilon=\frac{H}{\xi}=H \sqrt{\frac{\omega}{v}}
$$

which is the result derived above, namely, Eq. (28) with $b=0$.

The FDT can also be used in a local form

$$
\mu\left(z, z^{\prime}, \omega\right)=\frac{1}{k_{B} T} \int_{0}^{\infty}\left\langle u(z, t) u\left(z^{\prime}, 0\right)\right\rangle e^{i \omega t} d t,
$$

where $\mu\left(z, z^{\prime} \omega\right)$ is a local complex admittance defined by

$$
\langle u(z, \omega)\rangle=\int_{0}^{H} d x^{\prime} \mu\left(z, z^{\prime}, \omega\right) F\left(z^{\prime}, \omega\right),
$$

and a similar relation upon swapping $z$ with $z^{\prime} \cdot \mu\left(z, z^{\prime} \omega\right)$ is thus the solution of

$$
\int_{0}^{H} d z^{\prime} \mu\left(z, z^{\prime}, \omega\right) F\left(z^{\prime}, \omega\right)=\mu(z, \omega)
$$

and with our notation $\tilde{C}\left(z, z^{\prime}, \omega\right)=k_{B} T \mu\left(z, z^{\prime}, i \omega\right)$. One can check that $\tilde{C}\left(z, z^{\prime}, \omega\right)$ found with the second method [Eq. (27)] provides a solution to Eq. (36).

Similar considerations apply to the cylindrical channel: again one can verify that $\mu\left(r, r^{\prime}, \omega\right)=\tilde{C}\left(r, r^{\prime}, \omega / i\right) / k_{B} T$ is the solution of the equation

$$
\int_{0}^{R} r^{\prime} d r^{\prime} \mu\left(r, r^{\prime}, \omega\right) F\left(r^{\prime}, \omega\right)=\mu(r, \omega),
$$

where the complex admittance $\mu(r, \omega)$ is

$$
\mu(r, \omega)=\frac{i}{m \omega}\left[1-\frac{I_{0}\left(\frac{r}{\xi}\right)}{I_{0}\left(\frac{R}{\xi}\right)+\frac{b}{R} I_{1}\left(\frac{R}{\xi}\right)}\right] .
$$

\section{Friction and random forces}

From the velocity correlation function $C(t)$ of the c.m., one can characterize the friction force $\mathcal{F}$ exerted on the channel, as well as the random force $F_{R}$ that would appear in a generalized Langevin equation. For completeness, those expressions are reported here.

If $\mathcal{S}=2 \pi R L$ is the surface of the cylinder, the friction force exerted by the fluid on the wall is $\mathcal{F}(t)=\left.\mathcal{S} \eta \partial_{r} u(r, t)\right|_{r=R}=$
$\mathcal{S} \eta / b u(R, t)$, and its correlation function has for time Laplace transform

$$
\tilde{C}_{\mathcal{F}}(\omega)=\frac{\mathcal{S} k_{B} T \eta / b}{1+\frac{b}{R} \frac{\epsilon I_{1}(\epsilon)}{I_{0}(\epsilon)}}, \quad \epsilon=R \sqrt{\frac{\omega}{v}} .
$$

In the limit $\omega \rightarrow 0$, one gets

$$
\frac{1}{\mathcal{S} k_{B} T} \int_{0}^{\infty}\langle\mathcal{F}(t) \mathcal{F}(0)\rangle d t=\frac{\eta}{b},
$$

which is the expected result [62]. For the slit case, the righthand side is $2 \eta /[b(1+2 b / L)]$.

As regards the random force $F_{R}(t)$, the Laplace transform of its correlation function $\tilde{C}_{F_{R}}(\omega)$, and the memory kernel, can be deduced from $\tilde{C}(\omega)$ using the relation

$$
\frac{m \omega}{k_{B} T} \tilde{C}(\omega)\left[1+\frac{\tilde{C}_{F_{R}}(\omega)}{m \omega k_{B} T}\right]=1 .
$$

\section{DISCUSSION}

For conciseness, we focus on the case of water inside a cylindrical channel, unless otherwise mentioned.

\section{A. Diffusion coefficient and end effects}

Two main features of the c.m. motion observed in simulation are borne out by the calculations of Sec. III. First, the Einstein relation $D=\mu k_{B} T$ for the fluid c.m. motion holds. Indeed, for a fluid subject to gravity $g$, the total force is $F=$ $\pi R^{2} L \rho g$, the mean velocity of the resulting Poiseuille flow is $\bar{v}=(1+4 b / R) g R^{2} /(8 v)$, yielding the mobility $\mu=\bar{v} / F=$ $(1+4 b / R) /(8 \pi \eta L)$, in agreement with Eq. (33). Second, the c.m. motion is non-Markovian. As indicated by Eq. (32), the correlation function $C(t)$ differs from the pure exponential that is obtained for the simple (memoryless) Langevin equation. In the long time limit, though, and consistent with Fig. 6, a simple exponential decay is recovered, whose characteristic time is, up to a numerical prefactor, $R^{2} / \nu$, the time required by the momentum to diffuse over the channel cross section.

Let us now take the formula for $D$ at face value and estimate its order of magnitude for water in a 100-nm-long channel. With zero slip, the diffusion coefficient is $D \simeq$ $2 \times 10^{-12} \mathrm{~m}^{2} \mathrm{~s}^{-1}$, a value that would be expected for a colloid with $100-\mathrm{nm}$ radius or a 5000-bp DNA molecule. A slip length in the nanometric range, as found on hydrophilic surfaces, will induce only a small change to the above estimate. On the other hand, a significant effect is expected for carbon nanotubes that conjugate large slip length with small radius [66]. For instance, if $R=3.5 \mathrm{~nm}$ and $b=120 \mathrm{~nm}$ [22], one finds $4 b / R \simeq 137$ and an amplification factor of two orders of magnitude. In a smaller tube, with $R=0.5 \mathrm{~nm}$ and $b=500 \mathrm{~nm}$, this amplification factor reaches $4 \times 10^{3}$. For a tube length $L=$ $10 \mathrm{~nm}$, this would yield $D \simeq 6.4 \times 10^{-8} \mathrm{~m}^{2} \mathrm{~s}^{-1}$, which is larger than the diffusion coefficient for small ions in water.

While the above numbers apply to the periodic nanopore considered so far, real pores are connected to a reservoir at both ends, where velocity gradients always result in viscous losses. Such end effects can even be the dominant source of dissipation when slippage is high inside the channel, as is the case for carbon nanotubes $[67,68]$. A simple way to account 
for end friction is to write [69]

$$
\xi=\xi_{L}+\xi_{\text {end }} \text {. }
$$

Here, $\xi_{L}=k_{B} T / D$ is the friction coefficient for the fluid inside the channel, which was computed above. $\xi_{\text {end }}=3 \pi^{2} \eta R$ is the friction for a flow through a circular aperture in an infinitely thin wall as computed in Refs. [70,71]. Although approximate, Eq. (38) is numerically accurate [69], at least for the no-slip boundary condition. ${ }^{14}$ The corrected diffusion coefficient is therefore $\mathcal{D}=k_{B} T /\left(\xi_{L}+\xi_{\text {end }}\right)$, and reads as

$$
\mathcal{D}=\frac{k_{B} T}{\frac{1}{1+4 b / R} \times 8 \pi \eta L+3 \pi^{2} \eta R} .
$$

Depending on the relative values of $\xi_{\text {end }}$ and $\xi_{L}$, two regimes emerge separated by the crossover length $L_{c}=\frac{3 \pi}{8}(R+4 b)$. If $L>L_{c}$, which occurs for long tubes or small slip, the end effects can be neglected and the c.m. diffusion coefficient is that of a particle with effective size $L$ and slippage corrections. If $L<L_{c}$, as for short channel or large slip length, end effects dominate and the diffusion coefficient reduces to $\mathcal{D} \simeq$ $k_{B} T /\left(3 \pi^{2} \eta R\right)$ : the c.m. diffuses like a particle with effective diameter $\mathcal{R}=\pi R / 2$. For the nanotube $0.5 \mathrm{~nm}$ in radius considered above, the diffusion coefficient is now decreased by two orders of magnitude, down to $\mathcal{D} \simeq 3 \times 10^{-10} \mathrm{~m}^{2} \mathrm{~s}^{-1}$. Yet it remains significant: using the Stokes-Einstein formula, it corresponds to a particle with radius $0.75 \mathrm{~nm}$.

\section{B. Enhanced diffusion of a suspended particle}

We now discuss an experimental signature of the fluctuating motion of the fluid: with respect to the no-fluctuation case, a particle immersed within the channel may exhibit an enhanced diffusion. For concreteness, consider a particle of radius $a \lesssim$ $R$, whose motion is essentially one dimensional. The particle displacement is assumed to result from the superposition of two processes: (i) the motion of the particle with respect to that of the fluid and (ii) the global motion of the fluid. Both motions are diffusive with coefficients $D_{0}$ and $\mathcal{D}$, respectively, which yield for the particle diffusion an apparent coefficient $D=D_{0}+\mathcal{D}$.

Whether the enhancement is significant depends on the relative magnitude of $D_{0}$ and $\mathcal{D}$. The diffusion coefficient $D_{0}$ of a spherical particle moving through a cylindrical pipe was first examined by Faxén, and subsequently generalized to other situations [71]. In particular, the Renkin formula, which includes both hydrodynamic friction and steric hindrance at the entrance [72], reads as $D_{0}=D_{\mathrm{SE}} \phi(a / R)$ with $D_{\mathrm{SE}}=k_{B} T /(6 \pi \eta a)$ the Stokes-Einstein formula, and a hindrance correction factor $\phi(\varepsilon) \simeq(1-\varepsilon)^{2}\left(1-2.104 \varepsilon+2.09 \varepsilon^{3}-0.95 \varepsilon^{5}+\cdots\right)$.

Let us estimate the ratio $\mathcal{D} / D_{0}$ in two limiting cases. On the one hand, for $L \gg L_{c}$, the diffusion coefficient is approximately $\mathcal{D} \simeq k_{B} T /(8 \pi \eta L)$, which for large $L$ is much smaller than $D_{0}$. Yet, in a short tube whose radius is barely larger than the particle $(a \simeq R)$, the $\mathcal{D} / D_{0}$ ratio can be of order unity. To be specific, for a particle $1 \mathrm{~nm}$ in radius confined within a tube $10 \mathrm{~nm}$ in length and $1.5 \mathrm{~nm}$ in radius, the

\footnotetext{
${ }^{14}$ For perfect slip boundary condition, there is a small change in prefactor $\xi_{\text {end }} \approx 3.75 \pi^{2} \eta R$. Personal communication from L. Joly.
}

Faxén-Renkin formula gives $D_{0} \simeq 1.2 \times 10^{-11} \mathrm{~m}^{2} \mathrm{~s}^{-1}$, while $\mathcal{D} \simeq 1.6 \times 10^{-11} \mathrm{~m}^{2} \mathrm{~s}^{-1}$. On the other hand, the limit $L \ll$ $L_{c}$, where end effects dominate and $\mathcal{D} \simeq k_{B} T /\left(3 \pi^{2} \eta R\right)$, is relevant for carbon nanotubes and their large slip length. In this case, the Faxén-Renkin formula underestimates the diffusion coefficient. However, because of the necessary recirculation of the fluid around the particle inside the confining cylinder, $D_{0}$ never exceeds the bulk Stokes-Einstein prediction, even in the perfect slip limit [73]. As a consequence, one gets $\mathcal{D} / D_{0} \gtrsim$ $a / R$, which is again of order unity in strong confinement $a \simeq R$. In conclusion, we see that the fluctuation-induced motion of the fluid can strongly enhance the apparent diffusion coefficient of particles immersed in nanochannels.

Because it detects the passage of a particle in a channel connecting two reservoirs, a Coulter counter is a specific experimental setup that could demonstrate enhanced diffusion [74]. The ionic current, which is continuously monitored, exhibits a drop whenever a particle is present in the channel. ${ }^{15}$ The duration of the drop is simply the residence time of the particle within the channel. If there is no force (electrophoretic or mean flow) driving the particle, the distribution of residence time is controlled by the diffusion of the particle. An experimental realization has been presented in Ref. [75] for a nanotube $75 \mathrm{~nm}$ in radius. Significant effects of fluctuation-induced fluid motion are expected if the radius can be decreased down to a few nanometers.

\section{APPLICATION TO CHARGE TRANSPORT}

With the notable exception of carbon nanotubes, most artificial nanopores have charged walls, and an electric double layer at the solid-fluid interface [14], that may induce electrokinetic effects [76,77]. In such a situation, the thermal motion of the fluid induces a fluctuating electric current. In this section, we compute the corresponding contribution to the current power spectrum.

Simple assumptions are made on the charge distribution $\rho_{e}$. Discarding the possibility of fluctuations in ion distribution or surface charge [78,79], $\rho_{e}$ is taken as time independent. Furthermore, its spatial dependence is only transverse ( $z$ or $r$ coordinate), and it therefore remains unaffected by the fluid motion along the channel ( $x$ direction). The charge is thus passively transported by the fluid. In the general case where an applied electric field might be present, the total current in the slit channel is

$$
I_{T}(t)=e W \int_{0}^{H} d z\left[v_{+}(z, t) \rho_{+}(z)-v_{-}(z, t) \rho_{-}(z)\right] .
$$

Here, $e$ is the proton charge, $v_{ \pm}(z, t)=u(z, t) \pm \mu e E_{x}$ are the velocities of cations and anions, which are assumed monovalent and of equal mobility $\mu$, and $E_{x}$ is a constant electric field aligned with the channel. The total current can be separated in an "ion" contribution and a fluid contribution

$$
\begin{aligned}
I_{T}(t) & =I_{\text {ion }}(t)+I_{f}(t) \\
& =W \mu e E_{x} \int_{0}^{H} d z \rho_{T}(z)+e W \int_{0}^{H} d z u(z, t) \rho_{e}(z),
\end{aligned}
$$

\footnotetext{
${ }^{15}$ Here, we consider the low concentration situation where at most one particle is present in the channel at a given time.
} 
with $\rho_{T}=\rho_{+}+\rho_{-}$the total number density of ions and $\rho_{e}=$ $e\left(\rho_{+}-\rho_{-}\right)$the charge density. Now, the current correlation function is

$$
C_{I_{T}}(t)=\left\langle I_{T}(t) I_{T}(0)\right\rangle=C_{I_{f}}(t)+C_{I_{\text {ion }}}(t) .
$$

The cross-correlation term vanishes because $I_{f}$ and $I_{\text {ion }}$ are independent and of zero mean. In the following, we focus exclusively on $C_{I_{f}}$, the fluid contribution to the current correlation function. For clarity's sake, the $f$ subscript is dropped and $I_{f}(t)$ is denoted as $I(t)$. The correlation function $\tilde{C}_{I}(t)$ has for Laplace transform

$$
\tilde{C}_{I}(\omega)=W^{2} \iint_{0}^{H} \rho_{e}(z) \rho_{e}\left(z^{\prime}\right) \tilde{C}\left(z, z^{\prime}, \omega\right) d z d z^{\prime},
$$

which we attempt to evaluate below for several cases of charge distribution.

Knowing $\tilde{C}_{I}(\omega)$ gives access to the experimentally relevant quantity, namely, the power spectrum $S(\omega)=\tilde{C}(i \omega)+$ $\tilde{C}(-i \omega)=2 \operatorname{Re}[\tilde{C}(i \omega)]$. If the full expression $S(\omega)$ remains out of reach, one can nevertheless characterize the low and high frequency behaviors

$$
\begin{array}{ll}
\omega \rightarrow 0 & S(\omega)=2 D_{I}\left[1+f\left(\omega / \omega_{o}\right)\right], \\
\omega \rightarrow \infty & S(\omega)=\omega_{c}^{2} / \omega^{2}, \quad \omega_{c}^{2}=-2 C_{I}^{\prime}(0) / D_{I} .
\end{array}
$$

Here, we have introduced $D_{I}=\lim _{\omega \rightarrow 0} \tilde{C}_{I}(\omega)$, a function $f$ such that $f(0)=0$, and the characteristic and cutoff frequencies $\omega_{o}$ and $\omega_{c}$, that come out of low and high frequency expansion, respectively. ${ }^{16}$

\section{A. Current correlation function}

To specify the charge distribution, one introduces the surface charge $q_{s}=e \Sigma_{s}$ expressed in $\mathrm{C} \mathrm{m}^{-2}$, as well as the Bjerrum, Debye, and Gouy-Chapman lengths. For a solution of monovalent salt with concentration $c_{s}$ and with dielectric permittivity $\varepsilon$, the Bjerrum length is $l_{B}=e^{2} /\left(4 \pi \varepsilon k_{B} T\right)$, the Debye length is $\lambda=\left(8 \pi l_{B} c_{s}\right)^{-1 / 2}$, and the Gouy-Chapman length is $l=\left(2 \pi \Sigma_{s} l_{B}\right)^{-1}$. Finally, $\mathcal{A}$ denotes the crosssectional area of the pore and $\mathcal{P}$ is the "perimeter" of the double layer, namely, $2 W$ for the slit and $2 \pi R$ for the cylinder.

\section{Large channel approximation}

For an arbitrary charge distribution, we consider the limit of an infinitely large channel, that is, $H \rightarrow \infty .{ }^{17}$ In this case, $\tilde{C}_{I}$ can be approximated as

$$
\begin{aligned}
\tilde{C}_{I}(\omega) & =\frac{k_{B} T W}{\rho L} \iint_{0}^{\infty} \rho_{e}(z) \rho_{e}\left(z^{\prime}\right) g\left(z, z^{\prime}, \xi\right) d z d z^{\prime}, \\
g\left(z, z^{\prime}, \xi\right) & =\frac{\xi}{2}\left[e^{-\left|z-z^{\prime}\right| / \xi}-\frac{1-b / \xi}{1+b / \xi} e^{-\left(z+z^{\prime}\right) / \xi}\right],
\end{aligned}
$$

\footnotetext{
${ }^{16}$ Besides, in the high frequency limit, we have $\tilde{C}_{I}(\omega)=C_{I}(0) / \omega+$ $C_{I}^{\prime}(0) / \omega^{2}+O\left(\omega^{-3}\right)$, where $C_{I}(0)$ can be evaluated from Eq. (23), with the result $C_{I}(0)=\frac{k_{B} T W}{\rho L} \int_{0}^{H} \rho_{e}^{2}(z) d z$ for the slit and $C_{I}(0)=$ $\frac{k_{B} T 2 \pi}{\rho L} \int_{0}^{R} r \rho_{e}^{2}(r) d r$ for the cylinder.

${ }^{17}$ The case $R \rightarrow \infty$ is equivalent.
}

where $\xi=\sqrt{\nu / \omega}$ as before. Taking now the limit $\omega \rightarrow 0$ leads to

$$
\tilde{C}_{I}(\omega)=D_{I}\left[1-\sqrt{\omega / \omega_{o}}+O(\omega)\right] .
$$

$D_{I}$ is evaluated using $\lim _{\omega \rightarrow 0} g\left(z, z^{\prime}, \xi\right)=\min \left(z, z^{\prime}\right)+b$, with the result

$$
D_{I}=\frac{k_{B} T \mathcal{P}}{\eta L}\left(\varepsilon \mathcal{E}+b q_{s}^{2}\right),
$$

where $\mathcal{E}=\frac{\varepsilon}{2} \int V^{\prime}(z)^{2} d z$ is the electrostatic energy per unit length and unit width. The characteristic frequency is

$$
\omega_{o}=\frac{v}{\lambda^{2}} \frac{\left(1+\frac{2 b}{\lambda}\right)^{2}}{4\left(1+\frac{b}{\lambda}\right)^{4}},
$$

which for large $b / \lambda$ approaches $v / b^{2}$.

Finally, taking the inverse Laplace transform of Eq. (40) indicates that in the long time limit

$$
t \rightarrow \infty \quad C_{I}(t) \simeq C_{I}(0)\left(\frac{t}{t_{o}}\right)^{-3 / 2},
$$

with $t_{o}^{3 / 2}=M_{1}^{2} /\left(2 \sqrt{\pi} v^{3 / 2} M_{2}\right), M_{1}=\int_{0}^{\infty}(z+b) \rho_{e}(z) d z$, and $M_{2}=\int_{0}^{\infty} \rho_{e}^{2}(z) d z \cdot{ }^{18}$ Such behavior is the signature of a long time tail in the fluid velocity correlation.

\section{Debye-Hückel approximation}

The distribution of electric charge is now

$$
\begin{aligned}
& \rho_{e}(z)=\frac{q_{s}}{\lambda \sinh \left(\frac{H}{2 \lambda}\right)} \cosh \left(\frac{z-H / 2}{\lambda}\right), \\
& \rho_{e}(r)=\frac{q_{s}}{\lambda I_{1}\left(\frac{R}{\lambda}\right)} I_{0}\left(\frac{r}{\lambda}\right)
\end{aligned}
$$

for the slit and cylindrical channels, respectively. Analytical expressions for $\tilde{C}_{I}(\omega)$ in both geometries are available and reported in Appendix B. Because they are not straightforward to analyze, we focus on limiting behaviors and particular cases. For brevity, we consider primarily the cylindrical channel.

Small- $\omega$ behavior. An expansion of $\tilde{C}_{I}(\omega)$ for small $\omega$ yields

$$
\begin{aligned}
\tilde{C}_{I}(\omega) & =D_{I}\left[1-\frac{\omega}{\omega_{o}}+O\left(\omega^{2}\right)\right], \\
D_{I} & =\frac{k_{B} T q_{s}^{2} \pi R^{2}}{\eta L}\left[1+\frac{2 b}{R}-\frac{I_{0} I_{2}}{I_{1}^{2}}\right], \\
\omega_{o} & =\frac{v}{\lambda^{2}} \frac{1+\frac{2 b}{R}-\frac{I_{0} I_{2}}{I_{1}^{2}}}{\left(-1+\frac{b^{2}}{\lambda^{2}}-\frac{4 b}{R}\right)+\left(\frac{2 b}{\lambda}-\frac{4 \lambda}{R}\right) \frac{I_{0}}{I_{1}}+\frac{2 I_{0}^{2}}{I_{1}^{2}}}
\end{aligned}
$$

where the argument of Bessel functions is $R / \lambda$. The expression for $\omega_{o}$ simplifies in the following limits:

$$
\lambda \rightarrow \infty \quad \omega_{o}=\frac{v}{R^{2}} \frac{6\left(1+\frac{4 b}{R}\right)}{1+\frac{6 b}{R}+\frac{12 b^{2}}{R^{2}}},
$$

\footnotetext{
${ }^{18}$ If $\zeta_{\text {pot }}$ denotes the zeta potential, then $M_{1}=-\varepsilon V(0)+b q_{s}=$ $-\varepsilon \zeta_{\text {pot }}$.
} 


$$
\frac{b}{\lambda} \text { or } \frac{b}{R} \rightarrow \infty \quad \omega_{o}=\frac{2 v}{b R}
$$

Large- $\omega$ behavior. $C_{I}(0)$ and the cutoff frequency $\omega_{c}$ are given by

$$
\begin{aligned}
C_{I}(0) & =\frac{k_{B} T \pi q_{s}^{2}}{\rho L} \frac{R^{2}}{\lambda^{2}} \frac{I_{0}^{2}-I_{1}^{2}}{I_{1}^{2}}, \\
\omega_{c}^{2} & =\frac{v^{2}}{\lambda^{4}} \frac{\left(2 \lambda^{2}-b R\right) I_{0}^{2}+2 b \lambda I_{0} I_{1}+b R I_{1}^{2}}{-R I_{0}^{2}+2 \lambda I_{0} I_{1}+(2 b+R) I_{1}^{2}},
\end{aligned}
$$

where the argument of Bessel functions is $R / \lambda$. Moreover, one has in particular

$$
\begin{aligned}
& \lambda \rightarrow \infty \quad \omega_{c}=\frac{2 v}{b R}\left(1+\frac{R}{4 b}\right)^{-1 / 2}, \\
& R \rightarrow \infty \quad \omega_{c}=\frac{v}{\lambda^{2}}\left(\frac{1+2 \lambda / b}{1+2 b / \lambda}\right)^{1 / 2} .
\end{aligned}
$$

Limiting cases. The full expression for $\tilde{C}_{I}(\omega)$ takes simple forms in the three following limiting cases.

(i) Large double layer $(\lambda \rightarrow \infty)$. The charge distribution is homogeneous and one recovers the case of mass transport treated in Sec. III. Specifically, $\tilde{C}_{I}(\omega)=\left(q_{s} \mathcal{P}\right)^{2} \tilde{C}(\omega)$.

(ii) Thin double layer $(\lambda \rightarrow 0)$. The slit and cylinder results are, respectively,

$$
\begin{aligned}
& \tilde{C}_{I}(\omega)=\frac{D_{I}}{1+\frac{b}{H} \epsilon \tanh \left(\frac{\epsilon}{2}\right)}, \quad D_{I}=\frac{k_{B} T}{\eta L} \mathcal{P} q_{s}^{2} b, \\
& \tilde{C}_{I}(\omega)=\frac{D_{I}}{1+\frac{b}{R} \epsilon \frac{I_{1}(\epsilon)}{I_{0}(\epsilon)}} .
\end{aligned}
$$

Those expressions can be deduced from Eq. (37) by noting that for $\lambda \rightarrow 0, \rho_{e}(x) \rightarrow q_{s} \delta(r-R)$.

(iii) Large channel ( $H$ or $R \rightarrow \infty)$. The slit and cylinder geometry lead to the same result

$$
\begin{aligned}
\tilde{C}_{I}(\omega) & =D_{I} \frac{\xi^{2}[\lambda \xi+b(\lambda+2 \xi)]}{(\lambda+2 b)(\xi+b)(\xi+\lambda)^{2}}, \\
D_{I} & =\frac{k_{B} T q_{s}^{2} \mathcal{P}}{2 \eta L} \lambda\left(1+\frac{2 b}{\lambda}\right) .
\end{aligned}
$$

Moreover, it is possible to find the inverse Laplace transform of Eq. (47). The complete expression with arbitrary slip length $b$ is given in Appendix B. For the case of zero slip, the result for $C_{I}(t)$ reads as

$$
\begin{aligned}
C_{I}(t) & =C_{I}(0) h\left(\frac{t}{t_{o}}\right), \quad t_{o}=\frac{\lambda^{2}}{\pi^{1 / 3} v} \\
h(u) & =-2 \sqrt{u / \pi}+e^{u}(1+2 u) \operatorname{erfc}(\sqrt{u}) .
\end{aligned}
$$

One can check in particular that for large time, $h(t) \sim t^{-3 / 2}$, as shown above.

\section{Gouy-Chapman solution}

Since the Gouy-Chapman (GC) solution applies for a single surface, we consider only the case of a large channel, where the two double layers do not overlap. The GC solution for the electrostatic potential $V$ reads as

$$
V(z)=-\frac{2 k_{B} T}{e} \ln \left[\frac{1+\gamma e^{-z / \lambda}}{1-\gamma e^{-z / \lambda}}\right],
$$

where $\gamma$ is given by $\gamma=-l / \lambda+\sqrt{1+(l / \lambda)^{2}}$. The charge distribution is $\rho_{e}(z)=-\varepsilon V^{\prime \prime}(z)$. In the limit $\lambda / l \ll 1$ obtained for large salt concentration or small surface charge, the DebyeHückel solution is recovered. In the "no-salt limit" limit $\lambda / l \gg$ 1 , and assuming $z \ll \lambda$, one gets $V(z)=-\frac{2 k_{B} T}{e} \ln \frac{2 \lambda}{z+l}$, and an algebraic decay for $\rho_{e}(z)$.

Under the assumption $\gamma<1, D_{I}$ can be computed as

$$
D_{I}=\frac{k_{B} T q_{S}^{2} \mathcal{P}}{\eta L} l_{I}, \quad l_{I}=\gamma l\left[1+\frac{2 b}{\lambda\left(1-\gamma^{2}\right)}\right] .
$$

In the Debye-Hückel limit, $l_{I}=\lambda / 2+b$ while in the nosalt limit, $l_{I}=l+b$. With the full GC solution, no analytical expression could be found for $\tilde{C}_{I}(\omega)$.

\section{B. Discussion}

Using results on $D\left(z, z^{\prime}\right)$, a general formula for $D_{I}$ can actually be obtained:

$$
D_{I}=\frac{k_{B} T \mathcal{P}}{\eta L}\left(\varepsilon \mathcal{E}+b q_{s}^{2}\right),
$$

where $\mathcal{E}$ is defined in Eq. (41). This expression of $D_{I}$ can be recovered directly from Green-Kubo relations (or Nyquist relation in the context of electric current). On the one hand, the conductance, defined as $I=K\left[V_{x}(L)-V_{x}(0)\right]$, is written as the sum $K=K_{E O}+K_{E P}$ of the electro-osmotic and electrophoretic contributions. The Green-Kubo relation for the former reads as

$$
K_{E O}=\frac{1}{k_{B} T} \int_{0}^{\infty}\langle I(t) I(0)\rangle d t,
$$

that is with our notations $D_{I}=K_{E O} k_{B} T$. On the other hand, $K_{E O}$ is shown in Ref. [19] to be $\mathcal{P}\left(\varepsilon \mathcal{E}+b q_{s}^{2}\right) /(\eta L)$. Putting the two results together yields back Eq. (48). This shows that the contribution of the c.m. fluctuations to the conductance identifies with the electro-osmotic contribution.

Coming back to the total current $\tilde{C}_{I_{T}}(\omega)=\tilde{C}_{I}(\omega)+$ $\tilde{C}_{I_{\text {ion }}}(\omega)$, the same argument leads to $D_{I_{\text {ion }}}=\lim _{\omega \rightarrow 0} \tilde{C}_{I_{\text {ion }}}(\omega)=$ $k_{B} T K_{E P}$, where the electrophoretic contribution to conductance is [80]

$$
K_{E P}=\frac{\mu e^{2}}{L}\left[2 c_{s} \mathcal{A} \cosh \left(\frac{e V_{c}}{k_{B} T}\right)+\frac{\mathcal{P} \mathcal{E}}{2 k_{B} T}\right] .
$$

Here, $V_{c}=V(H / 2)$ is the potential at mid-height of the slit. In particular, if one assumes no overlap between double layers and $l \ll \lambda$, which holds in the low salt regime $c_{s} \rightarrow 0, K_{E P}$ simplifies to

$$
K_{E P}=\frac{\mu e^{2}}{L}\left(2 c_{s} \mathcal{A}+\mathcal{P} \Sigma_{s}\right),
$$

where the term in brackets is the total number of charges per unit length. To conclude, just as in the case of mass transport, the c.m. diffusive motion satisfies Einstein relation, the low frequency limit of the current spectrum $S(0)=2 D_{I}$ is connected to the electric conductance by the Nyquist relationship. 
All calculations presented above are valid for a periodic channel. In contrast to mass transport, it seems difficult to reintroduce end effects. Indeed, as discussed in Ref. [81], surface conduction has a deep effect on the current outside the nanopore, resulting in anomalously large pore conductance, and an apparent size much larger than the nanopore radius. It is thus likely that the formulas derived in this section apply only to long channels, when end effects can be neglected.

With this note of caution, we can nevertheless estimate typical orders of magnitude. For zero slip, the characteristic frequency is $v / R^{2}$, which for a radius in the nanometer range yields a value above the $\mathrm{GHz}$. Such large values are also obtained in the large slip limit $b \rightarrow \infty$, for which $\omega_{0}=2 v /(b R)$. This would suggest that in most experiments, the power spectrum originating in thermal motion of fluid will appear as a constant contribution; in such a case, only the low frequency value matters $\left[S(0) \approx 2 D_{I}\right]$. As regards $C_{I}(0)=\left\langle I^{2}\right\rangle$, taking $q_{s}=0.05 \mathrm{C} \mathrm{m}^{-2}$ and $\lambda=1 \mathrm{~nm}$ yields $\sqrt{\left\langle I^{2}\right\rangle}$ in the $\mathrm{nA}$ range. One can check that in order of magnitude $D_{I} \simeq\left\langle I^{2}\right\rangle 2 \pi / \omega_{o}$.

\section{CONCLUSION}

It is well known that a small particle immersed in a fluid undergoes Brownian motion. In this work, we have shown that, in a similar manner, a fluid confined at the nanoscale exhibits a thermally induced stochastic motion. This phenomenon is expected to be relevant for nanopores, biological and artificial alike. Our main outcome is a generic description for the fluctuations of the hydrodynamic degrees of freedom. It captures analytically the dependence on channel size and slip length, and provides a guide to gauge the relevance of fluctuation effects.

Whereas in this work, molecular dynamics simulations serve only to illustrate the phenomena at play, it remains to test our analytical predictions on specific, atomically detailed systems. Among them, the narrow carbon nanotubes appear of particular interest because large slip length should result in significant fluctuation effects. An alternate direction is to retain a description at the mesoscopic level, but to use numerical simulations that include fluctuations, such as lattice Boltzmann methods [82]. Because the thermal fluctuations can be switched on or off [83], one could unambiguously identify their contribution to transport. Other mesoscale approaches include dissipative particle dynamics [84] and stochastic rotational dynamics [85]. Rather than the simple geometries considered here, such methods would allow us to treat realistic pore shapes.

On the experimental side, the most testable of our predictions may be the enhanced diffusion of a particle within the nanopore, with an apparent coefficient that includes the fluid contribution. The effect should appear in channel with radius in the nanometer range, and could be evidenced using Coulter counter measurements or fluorescence correlation spectroscopy, if one can detect the signal from a particle inside the tube. We have taken the particle as pointlike, but a natural extension of this work is to treat the case of linear objects. One can still expect significant fluctuation effects when the translocating molecule has a size comparable to that of the pore, a case relevant for the 60-nucleotide-long DNA molecules used in Ref. [86].

As a final perspective, we remark that together with unbiased external forcing, the presence of fluctuations is the basic ingredient for ratchet effects and Brownian motors [87]. Direction motion of ions has been demonstrated for a charged conical pore subject to an oscillating electric, resulting in a ion pump [88]. Could asymmetrically shaped nanopores lead to rectification in the fluid motion? More generally, can we put the fluctuations at work? This question deserves further investigation.

\section{ACKNOWLEDGMENTS}

L.B. acknowledges support from ERC, AG-project Micromega. The authors thank L. Joly and A. Siria for fruitful discussions.

\section{APPENDIX A: FLUCTUATION RELATION}

Preliminary. Fluctuation relations characterize the statistical properties of fluctuations in nonequilibrium systems [89-91]. They have been developed for various situations, including deterministic or stochastic dynamics, and steady or transient regimes. As one of the few exact results valid arbitrarily far from equilibrium, they have been studied in a variety of contexts, both experimental and theoretical. Here, we show that the fluctuation relation takes a simple meaning for our nanofluidic system and verify numerically that it holds in our simulations.

In the following, only the steady state fluctuation relation (FR) is considered. As detailed in Ref. [92], FR have been proposed for two distinct quantities: the dissipation function $\Omega(t)$ and the phase space contraction rate $\Lambda(t)$. With the notation

$$
\bar{X}_{\tau}(t)=\frac{1}{\tau} \int_{t}^{t+\tau} X(s) d s,
$$

the $\Omega$-FR reads as

$$
\lim _{\tau \rightarrow \infty} \frac{1}{\tau} \ln \frac{\mathcal{P}\left(\bar{\Omega}_{\tau}=+u\right)}{\mathcal{P}\left(\bar{\Omega}_{\tau}=-u\right)}=u,
$$

while the $\Lambda$-FR is

$$
\lim _{\tau \rightarrow \infty} \frac{1}{\tau} \ln \frac{\mathcal{P}\left(-\bar{\Lambda}_{\tau}=+u\right)}{\mathcal{P}\left(-\bar{\Lambda}_{\tau}=-u\right)}=u .
$$

For isoenergetic dynamics, $\Omega(t)=-\Lambda(t)$ and therefore Eqs. (A1) and (A2) have identical meanings. Such equivalence does not hold for thermostated systems since in this case $\Omega(t)$ and $\Lambda(t)$ are not simply proportional to one another. More specifically, for a dissipative system in contact with a thermostat, the dissipation function is

$$
\Omega(t)=-\beta \mathbf{J}(t) V \cdot \mathbf{F},
$$

where $V$ is the volume of the system, $\mathbf{F}$ is the constant dissipative field, $\mathbf{J}$ is the corresponding dissipative flux, and $\beta=1 /\left(k_{B} T\right)$, with $T$ the temperature of the thermostat. On the other hand, the phase space contraction rate is

$$
\Lambda(t)=\beta \mathbf{J}(t) V \cdot \mathbf{F}+\beta \dot{\mathcal{H}},
$$


where $\mathcal{H}$ is the Hamiltonian. Note that those expressions hold whether all particles in the system, or only a subset of them, are thermostated [93].

Application. What are $\Omega$ and $\Lambda$ for our system and how are they related to the work and heat? Denoting by $F$ the total force acting on the fluid particles and by $\mathbf{v}$ the velocity of the fluid c.m., the dissipative flux is $\mathbf{J}=-\mathbf{v} / V$. If the motion is aligned with the driving force, then

$$
\Omega(t)=\beta v(t) F .
$$

The dissipation function is the power developed by the driving force, expressed in $k_{B} T$ units. We also have $\bar{\Omega}_{\tau}=\beta W_{\tau} / \tau$. On the other hand, Eq. (A4) yields

$$
\Lambda(t)=-\beta \dot{Q}(t),
$$

i.e., the phase space contraction rate is the rate of heat flow going from the thermostat to the system, expressed in $k_{B} T$ units [91]. As a consequence, $\bar{\Lambda}_{\tau}=-\beta Q_{\tau} / \tau$. From now on, we work with $W$ and $Q$, whose physical meaning is more intuitive

The fluctuation relations (A1) and (A2) can be expressed as

$$
\lim _{\tau \rightarrow \infty} \frac{k_{B} T}{\left\langle X_{\tau}\right\rangle} \ln \frac{\mathcal{P}\left(+u\left\langle X_{\tau}\right\rangle\right)}{\mathcal{P}\left(-u\left\langle X_{\tau}\right\rangle\right)}=u,
$$

where $\left\langle X_{\tau}\right\rangle$ denotes the average value for the quantity $X_{\tau}, X=$ $W$ for the $\Omega$-FR, and $X=Q$ for the $\Lambda$-FR. For convenience, we introduce the function

$$
f_{\tau}^{X}(u)=\frac{k_{B} T}{\left\langle X_{\tau}\right\rangle} \ln \frac{\mathcal{P}\left(+u\left\langle X_{\tau}\right\rangle\right)}{\mathcal{P}\left(-u\left\langle X_{\tau}\right\rangle\right)} .
$$

The fluctuation relation holds if $f_{\tau}^{X}(u) \rightarrow u$ for $\tau$ large enough. Note finally that the physical meaning of the $\Omega$-FR expressed by Eqs. (A1) is clear. Since $W_{\tau}=F x_{\tau}$, we have

$$
\lim _{\tau \rightarrow \infty} \ln \frac{\mathcal{P}\left(x_{\tau}=+u\right)}{\mathcal{P}\left(x_{\tau}=-u\right)}=\frac{u F}{k_{B} T},
$$

i.e., for sufficiently large time $\tau$, the relative probability of a c.m. displacement of magnitude $\left|x_{\tau}\right|$ in the forward and backward directions, decreases exponentially with $x_{\tau}$.

Figure 7 shows the function $f_{\tau}^{W}(u)$. For all times $\tau$ ranging from 0.1 to $100, f_{\tau}^{W}$ is linear, and in agreement with the FR, the slope approaches unity at large $\tau$. Those observations can be rationalized with the conclusions of Sec. II. A Gaussian distribution of mean $a$ and standard deviation $\sigma$,

$$
\mathcal{P}(x)=\frac{1}{\sqrt{2 \pi \sigma^{2}}} \exp \left[-\frac{(x-a)^{2}}{2 \sigma^{2}}\right],
$$

satisfies the relation

$$
\frac{\sigma^{2} /(2 a)}{a} \ln \frac{\mathcal{P}(+u a)}{\mathcal{P}(-u a)}=u,
$$

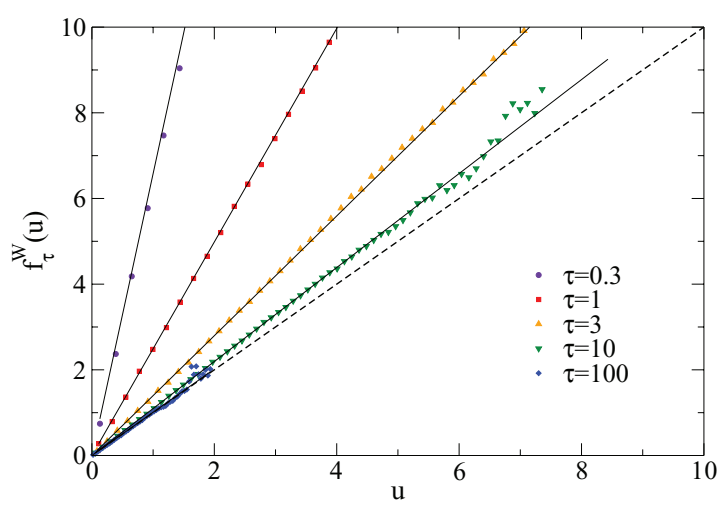

FIG. 7. (Color online) Test of the fluctuation relation. The lines are the predictions from Eq. (A12).

which is similar to the fluctuation relation [Eq. (A7)], if $\sigma^{2} /(2 a)$ can be identified with $k_{B} T$. As shown above, the distribution of displacement $\mathcal{P}\left(x_{\tau}\right)$ is a Gaussian with mean $a=\left\langle x_{\tau}\right\rangle=\tau \bar{v}$ and variance given by Eq. (1), i.e., $\left\langle x_{\tau}^{2}\right\rangle=$ $2 D \tau \alpha_{\tau}$, where $\alpha_{\tau}=1-\frac{1-e^{-\gamma \tau}}{\gamma \tau}$. Putting all this together yields

$$
\frac{D \alpha_{\tau} / \bar{v}}{\left\langle x_{\tau}\right\rangle} \ln \frac{\mathcal{P}\left(+u\left\langle x_{\tau}\right\rangle\right)}{\mathcal{P}\left(-u\left\langle x_{\tau}\right\rangle\right)}=u .
$$

Now, using the Einstein relation $D=\mu k_{B} T$, and $\left\langle W_{\tau}\right\rangle=$ $F\left\langle x_{\tau}\right\rangle$, Eq. (A11) can be rewritten as

$$
f_{\tau}^{W}(u)=\frac{k_{B} T}{\left\langle W_{\tau}\right\rangle} \ln \frac{\mathcal{P}\left(+u\left\langle W_{\tau}\right\rangle\right)}{\mathcal{P}\left(-u\left\langle W_{\tau}\right\rangle\right)}=\frac{u}{\alpha_{\tau}} .
$$

This expression gives an adequate description of the simulation data, as can be seen in Fig. 7. For large $\tau, \alpha_{\tau} \rightarrow 1$ and one recovers the fluctuation relation for the work.

As regards the distribution of heat $\mathcal{P}\left(Q_{\tau}\right)$, one finds that they are also Gaussian for all $\tau$ considered. However, in the range of time interval $\tau$ studied here, the FR for the heat is clearly not satisfied. This is not surprising since (i) the $\Lambda$-FR does not apply to thermostated systems, and (ii) numerical tests on a thermostated Lorentz gas found that the $\Lambda$-FR is satisfied far from equilibrium, but does not hold close to equilibrium [94].

\section{APPENDIX B: LONG FORMULAS}

Here, we report exact formulas obtained in the case of a Debye-Hückel double layer. The time Laplace transform of the current can be computed in both geometries. For the slit case,

$$
\begin{aligned}
\tilde{C}_{I}(\omega)= & \frac{k_{B} T q_{s}^{2} W}{\rho L \omega} \frac{\operatorname{csch}^{2}\left(\frac{H}{2 \lambda}\right)\left[b \cosh \left(\frac{H}{2 \xi}\right)+\xi \sinh \left(\frac{H}{2 \xi}\right)\right]}{\left(\lambda^{2}-\xi^{2}\right)^{2}\left[\left(b^{2}+\xi^{2}\right) \sinh \left(\frac{H}{\xi}\right)+2 b \xi \cosh \left(\frac{H}{\xi}\right)\right]}\left\{\xi \operatorname { c o s h } ( \frac { H } { 2 \xi } ) \left[2 b \xi^{2} \cosh \left(\frac{H}{\lambda}\right)-2 b \xi^{2}\right.\right. \\
& \left.+\lambda\left(\lambda^{2}+\xi^{2}\right) \sinh \left(\frac{H}{\lambda}\right)+H \lambda^{2}-H \xi^{2}\right]+\sinh \left(\frac{H}{2 \xi}\right)\left[b \lambda\left(\lambda^{2}-3 \xi^{2}\right) \sinh \left(\frac{H}{\lambda}\right)+b H \lambda^{2}-b H \xi^{2}\right. \\
& \left.\left.-2 \lambda^{2} \xi^{2} \cosh \left(\frac{H}{\lambda}\right)-2 \lambda^{2} \xi^{2}\right]\right\} .
\end{aligned}
$$


For the cylindrical case,

$$
\begin{aligned}
& \tilde{C}_{I}(\omega) \\
& =\frac{k_{B} T q_{s}^{2} \pi R}{\rho L \omega} \frac{R\left(\lambda^{2}-\xi^{2}\right)\left[b I_{1}\left(\frac{R}{\xi}\right)+\xi I_{0}\left(\frac{R}{\xi}\right)\right]\left[I_{0}^{2}\left(\frac{R}{\lambda}\right)-I_{1}^{2}\left(\frac{R}{\lambda}\right)\right]+2 \xi^{2}\left[b I_{1}\left(\frac{R}{\lambda}\right)+\lambda I_{0}\left(\frac{R}{\lambda}\right)\right]\left[\xi I_{1}\left(\frac{R}{\lambda}\right) I_{0}\left(\frac{R}{\xi}\right)-\lambda I_{0}\left(\frac{R}{\lambda}\right) I_{1}\left(\frac{R}{\xi}\right)\right]}{\left(\lambda^{2}-\xi^{2}\right)^{2} I_{1}\left(\frac{R}{\lambda}\right)^{2}\left[b I_{1}\left(\frac{R}{\xi}\right)+\xi I_{0}\left(\frac{R}{\xi}\right)\right]} .
\end{aligned}
$$

In the large channel approximation, the current correlation function $C_{I}(t)$ reads as

$$
\begin{aligned}
C_{I}(t) & =C_{I}(0) h\left(t / t_{c}\right), \quad t_{c}=\pi^{-1 / 3}(1+b / \lambda)^{4 / 3} \lambda^{2} / \nu, \quad C_{I}(0)=\frac{k_{B} T q_{s}^{2} \mathcal{P}}{2 \rho L \lambda}, \\
h(u) & =\frac{2 \sqrt{u}(b-\lambda)(b+\lambda)+\sqrt{\pi}\left[-e^{u}\left[b^{2}(-1+2 u)-(1+2 u) \lambda^{2}\right] \operatorname{erfc}(\sqrt{u})-2 b \lambda e^{\frac{u \lambda^{2}}{b^{2}}} \operatorname{erfc}\left(\frac{\sqrt{u} \lambda}{b}\right)\right]}{\sqrt{\pi}(b-\lambda)^{2}} .
\end{aligned}
$$

[1] B. Hille, Ionic Channels of Excitable Membranes (Sinauer Associates, Sunderland, MA, 1992).

[2] Aquaporins, edited by S. Hohmann, S. Nielsen, and P. Agre (Academic, New York, 2001).

[3] R. Baker, Membrane Technology and Applications (Wiley, Chichester, 2004).

[4] C. C. Striemer, T. R. Gaborski, J. L. McGrath, and P. M. Fauchet, Nature (London) 445, 749 (2007).

[5] S. Karan, S. Samitsu, X. Peng, K. Kurashima, and I. Ichinose, Science 335, 444 (2012).

[6] J. Li, D. Stein, C. McMullan, D. Branton, M. J. Aziz, and J. A. Golovchenko, Nature (London) 412, 166 (2001).

[7] A. J. Storm, J. H. Chen, X. S. Ling, H. W. Zandbergen, and C. Dekker, Nat. Mater. 2, 537 (2003).

[8] C. Dekker, Nat. Nanotechnol. 2, 209 (2007).

[9] M. Fyta, S. Melchionna, S. Succi, and E. Kaxiras, Phys. Rev. E 78, 036704 (2008).

[10] M. Fyta, S. Melchionna, and S. Succi, J. Polym. Sci., Part B: Polym. Phys. 49, 985 (2011).

[11] M. Majumder, N. Chopra, R. Andrews, and B. Hinds, Nature (London) 438, 43 (2005).

[12] J. K. Holt, H. G. Park, Y. Wang, M. Stadermann, A. B. Artyukhin, C. P. Grigoropoulos, A. Noy, and O. Bakajin, Science 312, 1034 (2006).

[13] J. Holt, Adv. Mater. 21, 3542 (2009).

[14] A. Siria, P. Poncharal, A.-L. Biance, R. Fulcrand, X. Blase, S. T. Purcell, and L. Bocquet, Nature (London) 494, 455 (2013).

[15] T. Squires and S. Quake, Rev. Mod. Phys. 77, 977 (2005).

[16] G. M. Whitesides, Nature (London) 442, 368 (2006).

[17] R. Schoch, J. Han, and P. Renaud, Rev. Mod. Phys. 80, 839 (2008).

[18] W. Sparreboom, A. van Den Berg, and J. Eijkel, Nat. Nanotechnol. 4, 713 (2009).

[19] L. Bocquet and E. Charlaix, Chem. Soc. Rev. 39, 1073 (2010).

[20] W. Sparreboom, A. van Den Berg, and J. C. T. Eijkel, New J. Phys. 12, 015004 (2010).

[21] D. J. Bonthuis, S. Gekle, and R. R. Netz, Langmuir 28, 7679 (2012).

[22] K. Falk, F. Sedlmeier, L. Joly, R. R. Netz, and L. Bocquet, Nano Lett. 10, 4067 (2010).

[23] D. J. Bonthuis, D. Horinek, L. Bocquet, and R. R. Netz, Phys. Rev. Lett. 103, 144503 (2009).
[24] J. S. Hansen, J. C. Dyre, P. J. Daivis, B. D. Todd, and H. Bruus, Phys. Rev. E 84, 036311 (2011).

[25] B. U. Felderhof, Phys. Fluids 23, 092002 (2011).

[26] J.-L. Barrat and J.-P. Hansen, Basic Concepts for Simple and Complex Liquids (Cambridge University Press, Cambridge, UK, 2003).

[27] A. Berezhkovskii and G. Hummer, Phys. Rev. Lett. 89, 064503 (2002).

[28] H. Fang, R. Wan, X. Gong, H. Lu, and S. Li, J. Phys. D: Appl. Phys. 41, 103002 (2008).

[29] M. Moseler and U. Landmann, Science 289, 1165 (2000).

[30] J. Eggers, Phys. Rev. Lett. 89, 084502 (2002).

[31] B. Davidovitch, E. Moro, and H. A. Stone, Phys. Rev. Lett. 95, 244505 (2005).

[32] G. Grün, K. Mecke, and M. Rauscher, J. Stat. Phys. 122, 1261 (2006).

[33] R. Fetzer, M. Rauscher, R. Seemann, K. Jacobs, and K. Mecke, Phys. Rev. Lett. 99, 114503 (2007).

[34] R. M. M. Smeets, U. F. Keyser, N. H. Dekker, and C. Dekker, Proc. Natl. Acad. Sci. USA 105, 417 (2008).

[35] F. Detcheverry and L. Bocquet, Phys. Rev. Lett. 109, 024501 (2012).

[36] A. Kalra, S. Garde, and G. Hummer, Proc. Natl. Acad. Sci. USA 100, 10175 (2003).

[37] F. Zhu, E. Tajkhorshid, and K. Schulten, Phys. Rev. Lett. 93, 224501 (2004).

[38] S. Y. Liem, D. Brown, and J. H. R. Clarke, Phys. Rev. A 45, 3706 (1992).

[39] R. Khare, J. de Pablo, and A. Yethiraj, J. Chem. Phys. 107, 2589 (1997).

[40] B. H. Kim, A. Beskok, and T. Cagin, Microfluid. Nanofluid. 5, 551 (2008).

[41] S. Bernardi, B. D. Todd, and D. J. Searles, J. Chem. Phys. 132, 244706 (2010).

[42] L. Kantorovich, Phys. Rev. B 78, 094304 (2008).

[43] L. Kantorovich and N. Rompotis, Phys. Rev. B 78, 094305 (2008).

[44] D. Toton, C. D. Lorenz, N. Rompotis, N. Martsinovich, and L. Kantorovich, J. Phys.: Condens. Matter 22, 074205 (2010).

[45] S. Plimpton, J. Comput. Phys. 117, 1 (1995).

[46] K. P. Travis, B. D. Todd, and D. J. Evans, Phys. Rev. E 55, 4288 (1997) 
[47] G. Galliéro, C. Boned, and A. Baylaucq, Ind. Eng. Chem. Res. 44, 6963 (2005).

[48] A. Botan, B. Rotenberg, and V. Marry, J. Phys. Chem. C 115, 16109 (2011).

[49] D. C. Rapaport, Phys. Rev. A 36, 3288 (1987).

[50] J. Horbach and S. Succi, Phys. Rev. Lett. 96, 224503 (2006).

[51] N. Pottier, Nonequilibrium Statistical Physics (Oxford University Press, Oxford, 2009).

[52] L. P. Pitaevskii and E. Lifshitz, Statistical Physics, Part 2 (Butterworth-Heineman, Oxford, 2002).

[53] R. Fox and G. Uhlenbeck, Phys. Fluids 13, 1893 (1970).

[54] K. T. Mashiyama and H. Mori, J. Stat. Phys. 18, 385 (1978).

[55] R. Fox, J. Math. Phys. 19, 1993 (1978).

[56] J. de Zárate and J. Sengers, Hydrodynamic Fluctuations in Fluids and Fluid Mixtures (Elsevier, Amsterdam, 2006).

[57] A. Barabási and H. Stanley, Fractal Concepts in Surface Growth (Cambridge University Press, Cambridge, 1995).

[58] M. Rubinstein and R. Colby, Polymer Physics (Oxford University Press, Oxford, UK, 2003).

[59] B. Alder and T. Wainwright, Phys. Rev. Lett. 18, 988 (1967).

[60] R. Zwanzig and M. Bixon, Phys. Rev. A 2, 2005 (1970).

[61] M. Isobe, Phys. Rev. E 77, 021201 (2008).

[62] L. Bocquet and J. L. Barrat, Phys. Rev. E 49, 3079 (1994).

[63] D. Chandler, Introduction to Modern Statistical Mechanics (Oxford University Press, Oxford, 1987).

[64] H. Carslaw and J. Jaeger, Conduction of Heat in Solids (Oxford University Press, Oxford, 1959).

[65] J. R. Womersley, J. Physiol. 127, 553 (1955).

[66] S. K. Kannam, B. D. Todd, J. S. Hansen, and P. J. Daivis, J. Chem. Phys. 138, 094701 (2013).

[67] L. Joly, J. Chem. Phys. 135, 214705 (2011).

[68] T. B. Sisan and S. Lichter, Microfluid. Nanofluid. 11, 787 (2011).

[69] Z. Dagan, S. Weinbaum, and R. Pfeffer, J. Fluid Mech. 115, 505 (1982).

[70] R. A. Sampson, Philos. Trans. R. Soc. London A 182, 449 (1891).

[71] J. Happel and H. Brenner, Low Reynolds Number Hydrodynamics (Kluwer, Dordrecht, 1983).
[72] E. M. Renkin, J. Gen. Physiol. 38, 225 (1954).

[73] A. Saugey, L. Joly, C. Ybert, J. L. Barrat, and L. Bocquet, J. Phys.: Condens. Matter 17, S4075 (2005).

[74] R. R. Henriquez, T. Ito, L. Sun, and R. M. Crooks, Analyst 129, 478 (2004).

[75] L. Sun and R. M. Crooks, J. Am. Chem. Soc. 122, 12340 (2000).

[76] L.-J. Cheng and L. J. Guo, Chem. Soc. Rev. 39, 923 (2010).

[77] I. Pagonabarraga, B. Rotenberg, and D. Frenkel, Phys. Chem. Chem. Phys. 12, 9566 (2010).

[78] R. Netz, Eur. Phys. J. E 1, 203 (2000).

[79] D. P. Hoogerheide, S. Garaj, and J. A. Golovchenko, Phys. Rev. Lett. 102, 256804 (2009).

[80] S. Levine, J. R. Marriott, and K. Robinson, J. Chem. Soc., Faraday Trans. 2 71, 1 (1975).

[81] C. Lee, L. Joly, A. Siria, A.-L. Biance, R. Fulcrand, and L. Bocquet, Nano Lett. 12, 4037 (2012).

[82] H. Basagaoglu, S. Melchionna, S. Succi, and V. Yakhot, Europhys. Lett. 99, 64001 (2012).

[83] R. Adhikari, K. Stratford, M. E. Cates, and A. J. Wagner, Europhys. Lett. 71, 473 (2005).

[84] A. Vázquez-Quesada, M. Ellero, and P. Español, J. Chem. Phys. 130, 034901 (2009).

[85] G. Gompper, T. Ihle, D. Kroll, and R. Winkler, Adv. Polym. Sci. 221, 1 (2008).

[86] H. Liu, J. He, J. Tang, P. Pang, D. Cao, P. Krstic, S. Joseph, S. Lindsay, and C. Nuckolls, Science 327, 64 (2010).

[87] P. Hänggi and F. Marchesoni, Rev. Mod. Phys. 81, 387 (2009).

[88] Z. Siwy and A. Fuliński, Phys. Rev. Lett. 89, 198103 (2002).

[89] D. Evans and D. Searles, Adv. Phys. 51, 1529 (2002).

[90] R. Harris and G. Schütz, J. Stat. Mech. (2007) P07020.

[91] E. M. Sevick, R. Prabhakar, S. R. Williams, and D. J. Searles, Annu. Rev. Phys. Chem. 59, 603 (2008).

[92] D. J. Evans, D. J. Searles, and L. Rondoni, Phys. Rev. E 71, 056120 (2005).

[93] D. J. Searles, L. Rondoni, and D. J. Evans, J. Stat. Phys. 128, 1337 (2007).

[94] M. Dolowschiák and Z. Kovács, Phys. Rev. E 71, 025202 (2005). 